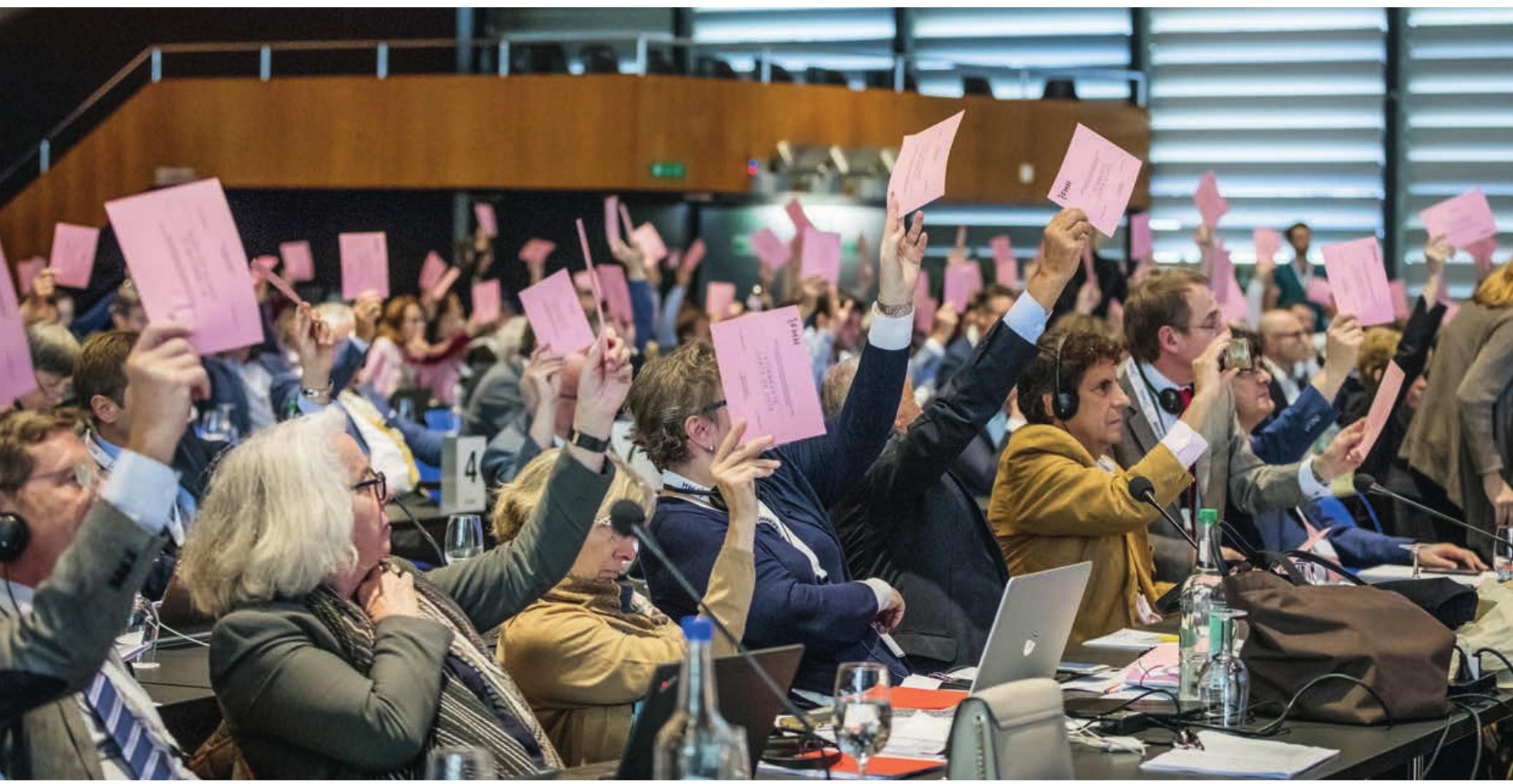

Die Delegierten stimmen über verschiedene Anträge ab. Sie haben unter anderem der aktualisierten Leistungsstruktur des ambulanten Tarifs und dem Weiterverfolgen einer gemeinsamen tarifpartnerschaftlichen Lösung mit überwältigendem Mehr zugestimmt.

\title{
Beschlussprotokoll der zweiten Ärztekammer 2018
}

\section{Monika Henzen}

Leiterin Abteilung Zentrales Sekretariat

Anmerkung der Protokollführerin: Aus Gründen der Lesbarkeit werden die Traktanden nach Massgabe der Nummerierung und nicht in der Reihenfolge der Diskussion aufgeführt.

\section{Begrüssung und Mitteilungen}

Nach Erreichen des Quorums eröffnet Dr. med. Jürg Schlup/Präsident FMH die zweite Ärztekammer 2018. Er heisst alle Delegierten wie insbesondere auch die neue Generalsekretärin, Dr. iur. Ursina Pally Hofmann herzlich willkommen. Sie hat am 1. Oktober 2018 die Leitung der operativen Geschäfte des Generalsekretariats der FMH in Personalunion mit der Leitung der Abtei- lung Rechtsdienst übernommen. Die 50-jährige Bündnerin ist promovierte Rechtsanwältin und Hebamme. Nach den üblichen organisatorischen Mitteilungen durch Ursina Pally Hofmann/Generalsekretärin FMH wird anschliessend das Büro bestellt. Dieses besteht aus dem Präsidenten, den Vizepräsidenten, der Generalsekretärin, RA Anne-Sylvie Thiébaud/Leiterin des Stimmund Wahlbüros, RA Julien Duruz/Stv. Leiter des Stimmund Wahlbüros sowie den nachfolgend mit $110 \mathrm{Ja}, 2$ Nein und 0 Enthaltung gewählten Stimmenzählenden: Alexander Zimmer, Christoph Helbling, Jean-Pierre Grillet, Florian Leupold, Hans-Anton Vogel, Franziska Zogg, Lucia Zürcher, Pierre Arnold, Karl-Olof Lövblad, Anja Zyska Cherix 


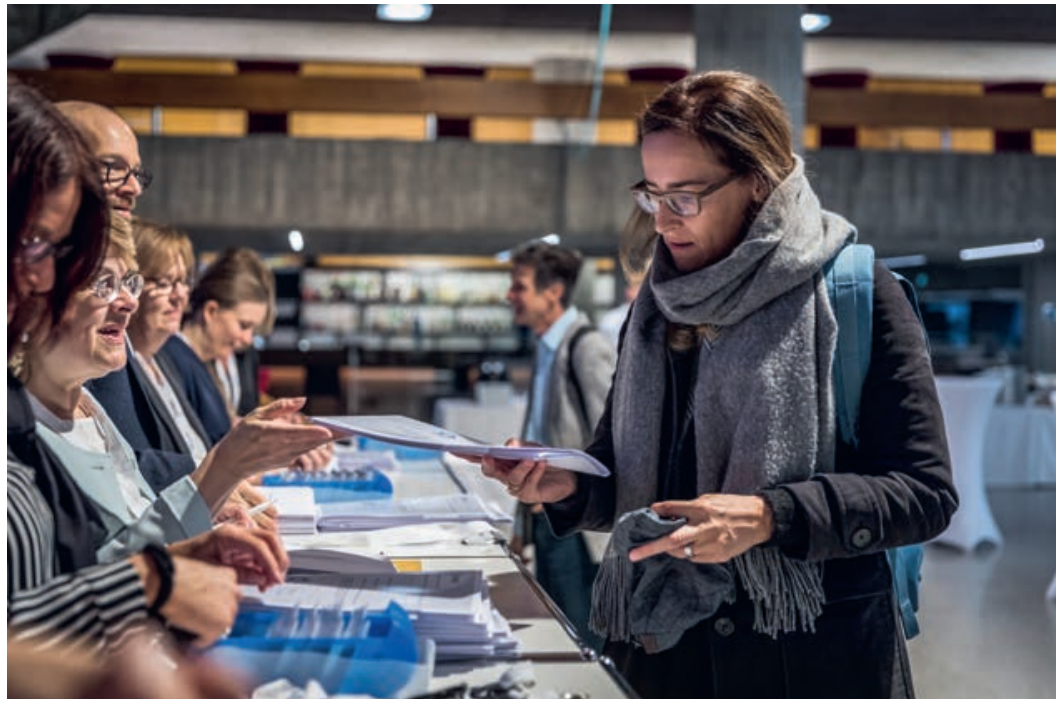

Die Teilnehmenden der Ärztekammer werden von den FMH-Mitarbeitenden herzlich begrüsst und nehmen die Unterlagen entgegen.
Beschluss:

Der Antrag wird mit 105 Ja, 3 Nein und 1 Enthaltung angenommen.

Art.11, Abs. 5 der GO FMH sieht vor, dass jeder Delegierte, jedes ZV-Mitglied und der Generalsekretär das Recht haben, Anträge zu den zur Diskussion stehenden Traktanden zu stellen. Anträge sind dem Vorsitzenden schriftlich vor, während der Sitzung oder ausnahmsweise mündlich einzureichen.

Ordnungsantrag 2 - Schriftliche Einreichung der Anträge:

Abänderungsvorschläge und Anträge sind dem Vorsitzenden schriftlich einzureichen. Mündliche Anträge werden nicht entgegengenommen und sind ungültig.

\section{Beschluss:}

Der Antrag wird mit 100 Ja, 23 Nein und 8 Enthaltungen angenommen.

\section{Eröffnungsreferat des Präsidenten}

Antrag:

Genehmigung der Traktandenliste.

Beschluss:

Die Traktandenliste wird einstimmig genehmigt. Gemäss Art. 11, Abs. 3 GO legt die ÄK zu Beginn jeder Sitzung die Zeit fest, nach deren Ablauf weder Beschlüsse gefasst noch Wahlen vollzogen werden dürfen. Der Präsident schlägt als Tagungsende 18.00 Uhr vor.

Antrag Festlegung Tagungsende:

Als Tagungsende wird 18.00 Uhr festgelegt. Nach 18.00 Uhr dürfen weder Beschlüsse gefasst noch Wahlen vollzogen werden.

Beschluss:

Der Antrag wird mit 110 Ja, 0 Nein und 3 Enthaltungen angenommen.

Der Vorsitzende stellt weiter zwei Ordnungsanträge zur Redezeitbeschränkung und zur Einreichung von Anträgen.

Gemäss Art. 11, Abs. 7 GO FMH kann auf Vorschlag aus der Kammer oder des Vorsitzenden die Redezeit allgemein beschränkt werden. Aufgrund der Traktanden und der zur Verfügung stehenden Zeit wird eine Redezeitbeschränkung von zwei Minuten beantragt.

Ordnungsantrag 1 - Redezeitbeschränkung:

Für die Behandlung der Traktanden der heutigen ÄK gilt eine Redezeitbeschränkung von 2 Minuten für Einzelredner. Keine Beschränkung gilt für den Präsidenten oder seinen Stellvertreter der jeweiligen stimm- oder antragsberechtigten Ärzteorganisation sowie für den Sprecher des ZV.
Santésuisse geht in ihrer im September 2018 veröffentlichten Studie "Ambulante Versorgungsstruktur und Bedarfsanalyse Schweiz» von einer Überversorgung von Ärzten in der Schweiz aus. Basierend auf ihren Projektionen fürs Jahr 2030 erwarten sie gesamtschweizerisch eine klare Tendenz zur Überversorgung. Die Realität sieht jedoch anders aus. Die vakanten Stellen haben in den letzten drei Jahren zugenommen. Am Jahresende 2017 gab es deutlich mehr offene Stellen für diplomiert worden sind. Es herrscht nach wie vor ein Ärztemangel.

Die ambulante Versorgung hat sich stark verändert. Neben der Zunahme von chronischen Krankheiten, multimorbider Patienten und der demografischen Entwicklung wirkt sich das zu kleine Angebot verfügbarer Studienplätze in den letzten 20 Jahren zusätzlich auf die heutige Situation aus. Die überbordende administrative Belastung wie auch seit neuestem die Limitation der Konsultationszeiten beschränken die verfügbare ärztliche Betreuungszeit.

Die Schweiz ist auf Ärztinnen und Ärzte mit auslänstützung dankbar. Trotzdem will der Bundesrat eine Verschärfung der Zulassungssteuerung. Die FMH fordert eine Zulassung nach Qualitätskriterien mit einer dreijährigen ärztlichen Tätigkeit in der für die Zulassung beantragten Fachdisziplin an einer anerkannten schweizerischen Weiterbildungsstätte sowie den Nachweis der erforderlichen Sprachkompetenz in der Sprache der Tätigkeitsregion. Ärzte (rund 1400), als im selben Jahr Ärzte hierzulande dischen Diplomen angewiesen und für diese Unter- 


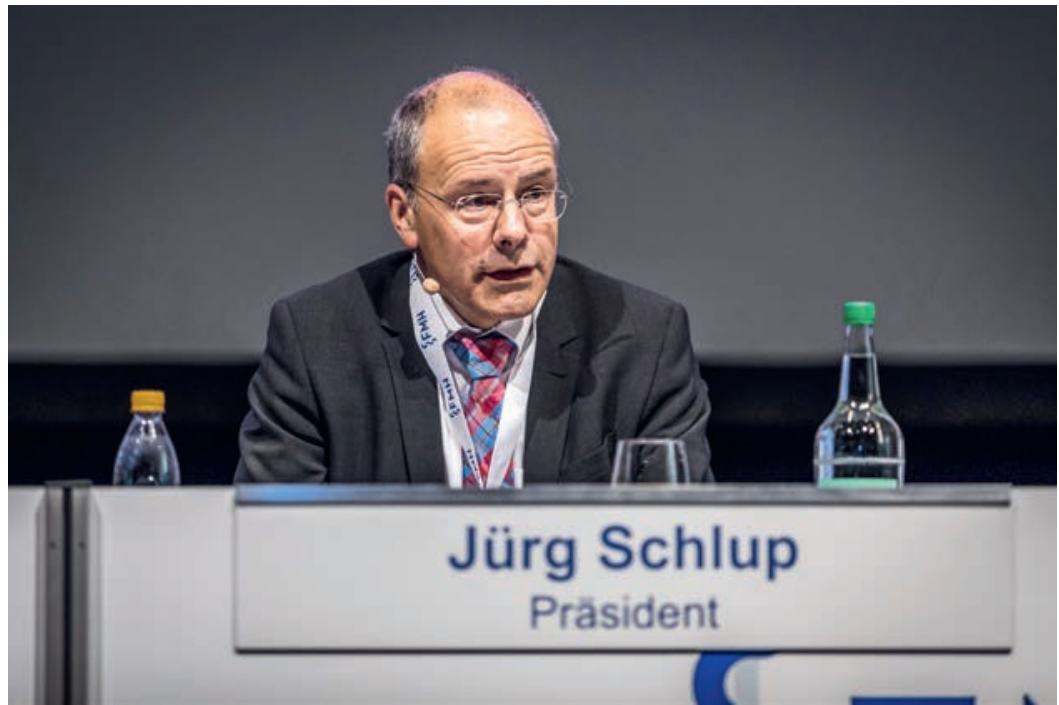

Der FMH-Präsident Dr. med. Jürg Schlup führt durch die Ärztekammer.

Gleichzeitig findet ein Kulturwandel in der Bevölkerung statt. Die Erwartungen der Patienten sind gestiegen. Der Versicherte wünscht heute eine zunehmend schnelle und umfassende Behandlung. Dagegen fordert die Politik Kostendämpfungsmassnahmen mit Zielvorgaben und Globalbudget. In keinem Land, welches über ein Globalbudget verfügt, wurden die Kosten gesenkt. Eine solche Massnahme führt vielmehr zu Wartezeiten, zu einer Zweiklassenmedizin sowie zu einem unverhältnismässig hohen administrativen Aufwand bei der Budgetverteilung. Wer das Globalbudget fordert, akzeptiert eine Zweiklassenmedizin. Die

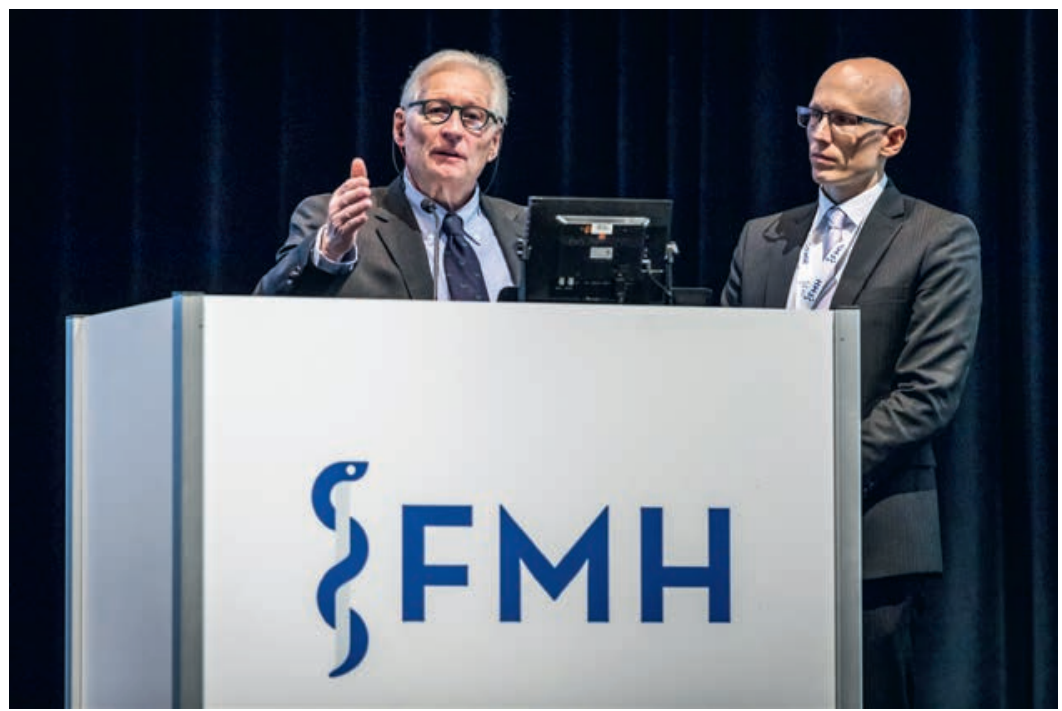

Dr. med. Urs Stoffel, Mitglied des FMH-Zentralvorstandes und Departementsverantwortlicher Ambulante Versorgung und Tarife, erläutert gemeinsam mit Patrick Müller, Abteilungsleiter Ambulante Versorgung und Tarife, die aktualisierte Leistungsstruktur des ambulanten Tarifs.
Ärzteschaft will keine Zweiklassenmedizin und keine Wartezeiten, sie will stattdessen einen Abbau der administrativen Belastung und eine einheitliche Finanzierung von ambulanten und stationären Leistungen das würde Kosten sparen. Und die Ärzteschaft will ein sachgerechtes Tarifsystem!

Die Blockade im Schweizer Gesundheitswesen hält weiter an und der politische Druck steigt. Die Ärzteschaft muss die politischen Herausforderungen als Chance nutzen. In demokratisch politischen Verhältnissen heisst Politik Auseinandersetzung. Wer keine politische Auseinandersetzung will, hat seine bessere Lösung bereits verloren. Die Politik ist nicht nur Schicksal - wie Napoleon sagte - sondern auch die Lösung. Um die Tarifautonomie zurückzugewinnen, appelliert der Vorsitzende an alle Delegierte, sich entschlossen und gemeinsam weiterhin für die laufende Revision des ambulanten Tarifs einzusetzen. Die FMH hat in jahrelanger Arbeit eine Leistungsstruktur entwickelt und erarbeitet, auf die sie stolz sein kann.

Der Ärzteverband braucht die politische Unterstützung in den eidgenössischen und kantonalen Parlamenten. Es empfiehlt sich, bei den eidgenössischen Wahlen 2019 jene Kandidierenden zu unterstützen, die sich einerseits für die Positionen der Ärzteschaft einsetzen - wie Ablehnung des Globalbudgets, Einstehen für die freie Arztwahl, die Tarifautonomie und eine Zulassungssteuerung über Qualitätskriterien und die andererseits Chancen auf eine Wahl bzw. Wiederwahl haben.

Der Präsident dankt allen Kolleginnen und Kollegen für ihre tägliche ärztliche Berufsarbeit im Dienste der Patienten und der Bevölkerung.

\section{Tarife}

\subsection{Ambulante Tarifrevision: Verabschiedung} verhandelte Leistungsstruktur inkl. Kostenmodelle und Taxpunkten

Dr. med. Urs Stoffel/ZV-Mitglied und Departementsverantwortlicher Ambulante Versorgung und Tarife präsentiert den aktuellen Stand der Revision des ambulanten Arzttarifs. Er erinnert an den Entscheid der ÄK vom 2. Mai 2018, bei dem die Delegierten den FMH-internen Vorschlag (Projekt TARCO) für die Leistungsstruktur einstimmig als wichtige Basis eines sachgerechten und betriebswirtschaftlichen berechneten Tarifs angenommen haben. Parallel zur Erarbeitung der Leistungsstruktur fanden gemäss Beschluss des Cockpits die Verhandlungen mit den Tarifpartnern innerhalb der ats-tms AG statt, um die Revision zeitgerecht zu beenden. Die Schwerpunkte der bisherigen Verhandlungen waren die Überprüfung gewisser Sparten im 


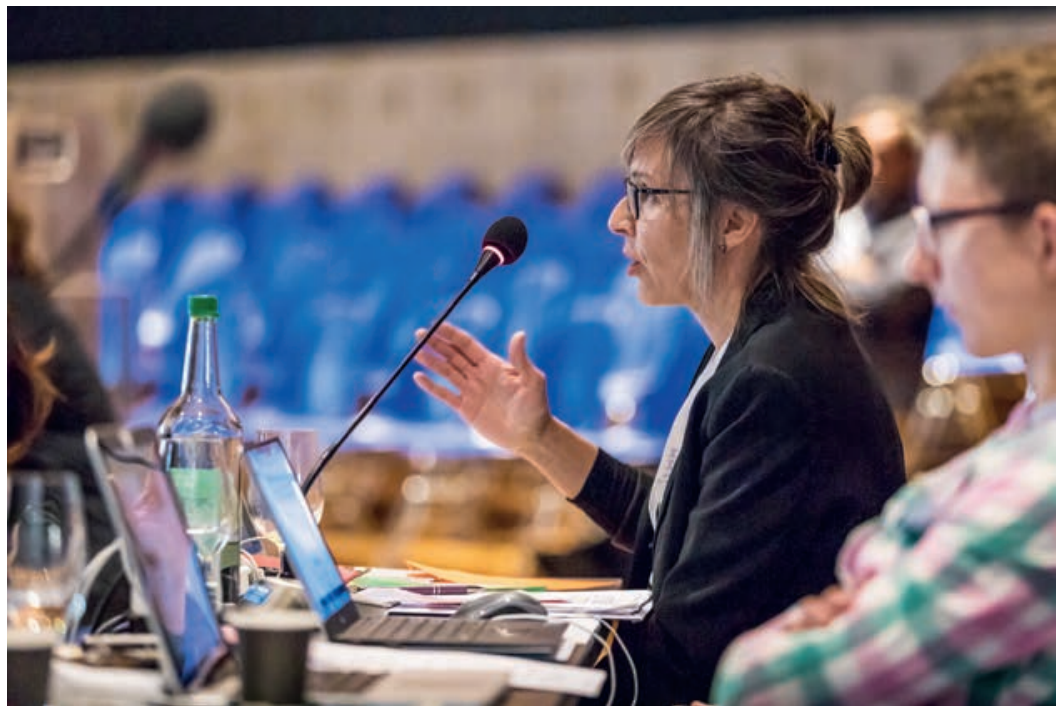

Die Delegierten vertreten ihre Basis- und Fachorganisationen in der Ärztekammer. Sie diskutieren angeregt zu den verschiedenen Themen und Anträgen: Hier die Präsentation des VSAO.
INFRA-Kostenmodell, die TARCO-Integration, die Mengen- und Zeitlimitationen, die Transcodierung sowie die Finalisierung der Leistungsstruktur. Die Resultate des Projektes TARCO konnten bei den gemeinsamen Verhandlungen mit den Tarifpartnern vollumfänglich eingebracht werden und wurden von diesen weitgehend übernommen.

Die Geschäftsstelle der ats-tms AG hat den VR am 16. August 2018 informiert, dass die verbleibende Zeit für die anstehenden offenen Aufgaben und Arbeiten zur vollständigen Finalisierung der Tarifstruktur (Leistungsstruktur, Kostenmodelle, Tarifführerschaft, Regelwerke und Verträge) bis Mitte September 2018 zu knapp und nicht realisierbar sei. Der VR der ats-tms AG hat aufgrund dieser neuen Situation eine Etappierung des Projekts beschlossen, damit gegenüber der Politik und Öffentlichkeit erste wichtige und gemeinsame Resultate kommuniziert werden können.

Die DV, welche am 6. September 2018 als erstes FMHOrgan über die Etappierung und den angepassten Zeitplan informiert wurde, war mit diesem Vorgehen einverstanden. Für die Delegierten ist es ein wichtiges Anliegen, bereits in diesem Jahr gegenüber der Öffentlichkeit und Politik erste Erfolge zu kommunizieren und dem Bundesrat ein gemeinsames erstes Paket (Leistungsstruktur inkl. Kostenmodelle und Taxpunkte) einzureichen. Zudem hat die DV den ZV Ende September 2017 ermächtigt zu prüfen, ob eine Kündigung des Aktionärbindungsvertrags der ats-tms AG sinnvoll sei. Im Rahmen dieser Austrittsverhandlungen konnte erreicht werden, dass die vertraglichen Grundlagen der ats-tms AG (Statuten und Aktionärsbindungsver- trag) grundlegend überarbeitet und einstimmig von der Generalversammlung im März 2018 verabschiedet wurden. Die deutliche Mehrheit der Anpassungsvorschläge der FMH wurden dabei umgesetzt. Dies ist als ein positiver Schritt der Tarifpartner zugunsten der FMH zu werten. Der Vorstand von H+ hat am 20. September 2018 einstimmig entschieden, den Aktionärbindungsvertrag der ats-tms AG vom 26. März 2018 zu kündigen. FMH, curafutura und MTK verbleiben jedoch weiterhin in der ats-tms AG.

Die Arbeiten der Etappe 1 an der jetzigen Leitungsstruktur inkl. Kostenmodellen und Taxpunkten sind abgeschlossen und werden substanziell nicht mehr modifiziert (vorbehalten Korrekturen Rechtsschreibung, Nachzug der Übersetzungen in Französisch und Italienisch, Ergänzungen in Leistungsgruppen, Kapitelund Tarifpositionsgliederung oder Korrekturen offensichtlicher Fehler). Nach Zustimmung der ÄK sollen die daraus resultierenden Resultate auch gegenüber der Öffentlichkeit und Politik als Teilerfolg kommuniziert werden. Mit Vorliegen der nun verhandelten Leistungsstruktur inkl. Taxpunkten ist das eigentliche Kernstück einer revidierten Tarifstruktur vorhanden. Ab Mitte November 2018 werden die noch offenen Themen betreffend Abrechnungsregeln und Transcodierung angegangen.

Unter Beizug der Fachgesellschaften werden in Etappe 2 kapitelweise die Abrechnungsregeln verhandelt. Parallel dazu werden die Transcodierung und die Simulationen aufbereitet. Die Arbeiten der Etappe 2 werden per März 2019 abgeschlossen sein. Dann liegt eine vollständig revidierte Tarifstruktur inkl. den erarbeiteten Abrechnungsregeln vor, die als Gesamtpaket mit den Grundverträgen und weiteren Konzepten (z.B. Plausibilisierung der Handlungsleistungen) nochmals den FMH-internen Gremien (DV und ÄK) vorgelegt wird. Die FMH hat im VR der ats-tms AG einen Antrag auf Anpassung der Bezeichnung des neuen Tarifvorschlags gestellt. Der vorläufige Arbeitstitel lautet «Leistungsstruktur ambulant». Zu erwähnen sind auch die umfangreichen Analysen und Berechnungen bezüglich der «Quantitativen Dignitäten» durch das Leitungsgremium AG Dignitäten. Das Leitungsgremium kam zum Schluss, dass sich die Individuelle Arztdignität (IAD) auf Basis heutiger Grundlagen nicht mehr rechtfertigen lässt. Das Cockpit folgte dem Antrag des Leitungsgremiums und beschloss, den IAD zum jetzigen Zeitpunkt nicht mehr weiter zu verhandeln.

Nach Urs Stoffel/ZV-Mitglied und Departementsverantwortlicher Ambulante Versorgung und Tarife ist die vorliegende Leistungsstruktur noch nicht perfekt und beinhaltet Kompromisse, die in den Verhandlungen einzugehen waren. Das kann für einzelne Fachgesell- 
schaften Probleme darstellen. Diese Diskussionen sollen jedoch in der Etappe 2 geführt werden.

Die interne Genehmigung der gesamten Tarifstruktur ist bei den FMH-Organen für April/Mai 2019 traktandiert. Die definitive Eingabe an den Bundesrat ist bis Mitte 2019 geplant, so dass der revidierte Tarif per 1. Januar 2020 eingeführt werden könnte.

Urs Stoffel/ZV-Mitglied und Departementsverantwortlicher Ambulante Versorgung und Tarife geht nochmals auf die bereits vom Präsidenten in seinem Eröffnungsreferat erwähnten Massnahmen zur Kostendämpfung ein. Diese werden einschneidend sein, wenn sich die Tarifpartner nicht einigen können. Eine der Massnahmen in der Vernehmlassung ist die Schaffung eines Nationalen ambulanten Tarifbüros. Der Bundesrat kann Grundsätze betreffend Form und Betrieb der Organisation auf Verordnungsstufe festlegen. Falls sich die Tarifpartner innert zwei Jahren ab Inkrafttreten nicht auf ein Tarifbüro einigen, wird der Bundesrat eingreifen und ein Nationales ambulantes Tarifbüro einsetzen.

In der anschliessenden Diskussion sprechen sich die Delegierten klar für eine Stärkung der ambulanten Versorgung aus. Voraussetzung dafür ist eine sachgerechte Tarifierung der ambulanten Leistungen. Die Eckwerte der Verhandlungen sind für die FMH definiert und gerade bei der Überarbeitung der entsprechenden Kapitel sind die einzelnen Fachgesellschaften gefordert.

Der ZV wird den Delegierten die definitive Tarifstruktur anlässlich der ÄK vom 9. Mai 2019 zur Genehmigung vorlegen.

Der Vorsitzende lässt über den Abstimmungsmodus abstimmen.

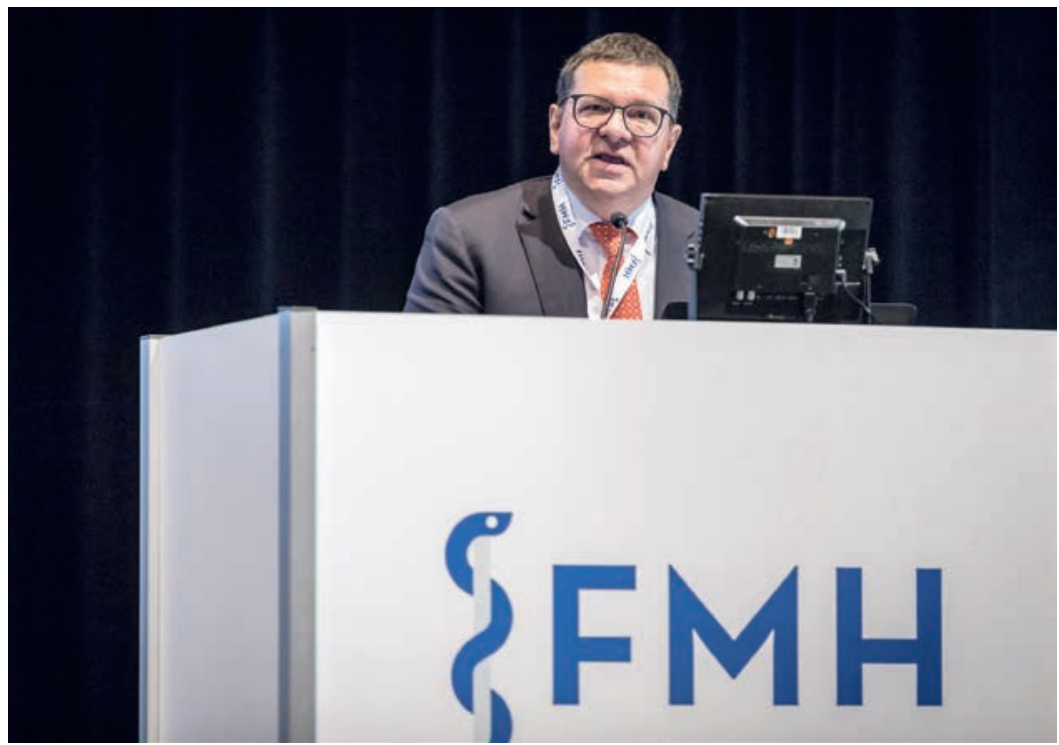

Reto Heiz, Leiter a.i. der Abteilung Finanzen und Verwaltung, legt das Budget 2019 der FMH und der GPK dar.
Antrag ZV:

Eine Gesamtabstimmung über alle drei Anträge versus drei einzelne Abstimmungen.

Beschluss:

Für eine Gesamtabstimmung über alle Anträge stimmen 150 Delegierte; für drei Abstimmungen 2 Delegierte. 1 Delegierter enthält sich der Stimme. Über die drei Anträge wird somit zusammen abgestimmt.

\section{Antrag ZV:}

Die ÄK stimmt

- zu, die vorliegende verhandelte Leistungsstruktur (Nomenklatur) inkl. Kostenmodellen und Taxpunkten (gemäss Tarifbrowser «Leistungsstruktur ambulant» V1.0) als Resultat der Etappe 1 der Verhandlungen abschliessend zu verabschieden.

- der Einreichung zur (Nomenklatur) inkl. Kostenmodellen und Taxpunkten (gemäss Tarifbrowser «Leistungsstruktur ambulant» V1.0) beim Bundesrat und BAG zur Vorprüfung zu.

- dem weiteren Vorgehen der Verhandlungen mit den Tarifpartnern mit der Erarbeitung der tarifarischen Abrechnungsregeln, Verträgen, Transcodierung und Simulationen als Resultat der Etappe $2 \mathrm{zu}$. Dieses Resultat wird den internen FMH-Genehmigungsorganen (DV und ÄK) im April/Mai 2019 vorgelegt.

Beschluss:

Die drei Anträge werden mit $158 \mathrm{Ja}, 1$ Nein und 3 Enthaltungen angenommen.

Urs Stoffel/ZV-Mitglied und Departementsverantwortlicher Ambulante Versorgung und Tarife dankt allen Beteiligten an diesem Projekt, aber ganz besonders seinem Team in Olten für den unermüdlichen und grossartigen Einsatz.

\section{Budgets 2019}

Jürg Schlup/Präsident FMH begrüsst Reto Heiz, der seit dem 1. September 2018 als Leiter a.i. der Abteilung Finanzen und Verwaltung im Generalsekretariat tätig ist. Vorgängig zur Budgetberatung informiert der Vorsitzende über die zwei zu diesem Traktandum eingereichten Anträge. Die SGAIM verlangt in ihrem am 4. Oktober 2018 eingereichten Antrag die Erhebung eines Sonderbeitrags für die Initiative "Ja zum Schutz der Kinder und Jugendlichen vor Tabakwerbung». Der VSAO will mit dem heute unterbreiteten Antrag die künftige Finanzierung von politischen Vorstössen klären. Der VSAO ist einverstanden, dass über seinen Antrag erst nach der Abstimmung zum konsolidierten Budget Beschluss gefasst wird. 


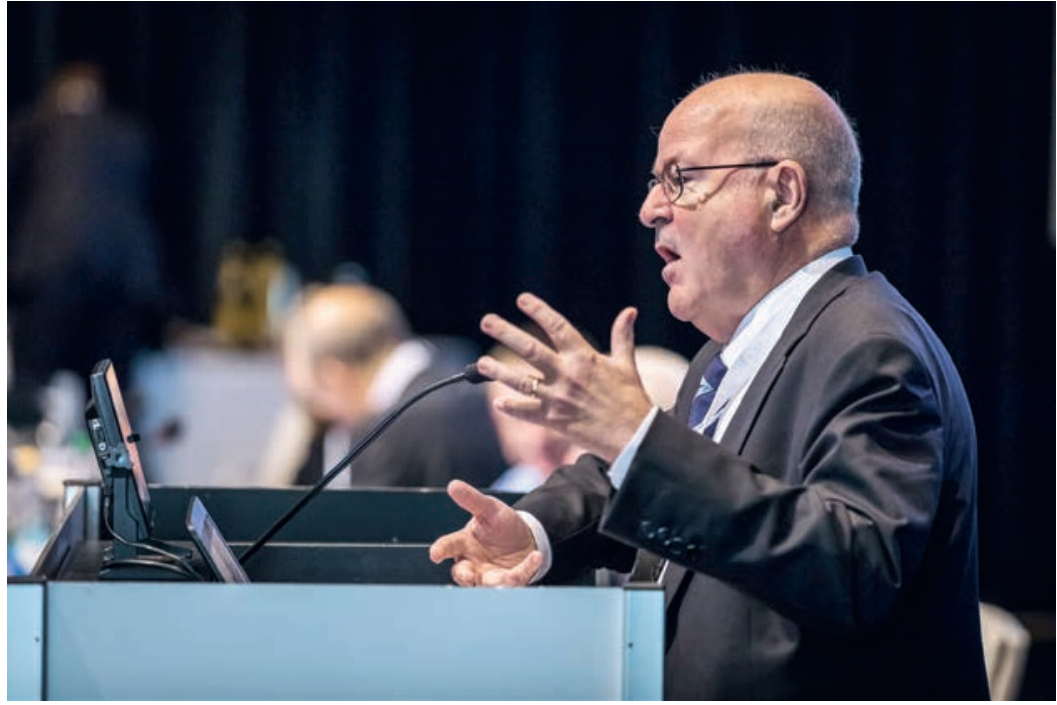

Der SIWF-Präsident, Dr. med. Werner Bauer, erläutert das Budget 2019 des SIWF.

\subsection{Budget 2019 SIWF}

Dr. med. Werner Bauer/Präsident SIWF berichtet über die abgeschlossene Akkreditierung 2018, in welcher die Experten eine positive Bilanz zur Qualität der Weiterbildungsgänge in der Humanmedizin gezogen haben. Unter der Verantwortung des SIWF und der Fachgesellschaften sind sie damit wieder für sieben Jahre akkreditiert. Das Prozedere ist aufwändig und auch kostenintensiv, da das SIWF die Kosten für die Fachgesellschaften übernimmt. Die wenig gemachten Auflagen und Empfehlungen werden mit den Fachgesellschaften nun besprochen. Bei der Fortbildung überlegt sich das Bundesamt für Gesundheit (BAG) ein vermehrtes regulatives Engagement und regt die Bildung einer entsprechenden Plattform-Themengruppe an, welche einen allfälligen Interventionsbedarf prüfen soll. Das BAG denkt z.B. an eine stärker strukturierte und kontrollierte Fortbildung und sogar an eine Re-Zertifizierung alle 10 Jahre. Das SIWF vertritt jedoch die Meinung, dass die Fortbildung auf der Selbstverantwortung der Ärzteschaft basiert und von ihr gestaltet werden muss. Die Plattform "Zukunft ärztliche Bildung» befasst sich zurzeit mit der Frage, wie häufig und warum die Weiterbildung länger geht als in den Weiterbildungsprogrammen vorgesehen. Zur Analyse dieser Problematik wurde eine Studie in Auftrag gegeben. Die Themengruppe «Koordination der ärztlichen Weiterbildung" hat ihre Arbeiten vorerst abgeschlossen. Das Schweizerische Gesundheitsobservatorium (Obsan) hat ein Modell zur Berechnung des zukünftigen Bestands und Bedarfs an Fachärztinnen und -ärzten in der Schweiz erarbeitet, das aber wegen einer noch nicht genügenden Datenlage der Optimierung bedarf, um wirklich aussagekräftig zu sein.
Christoph Hänggeli/Geschäftsführer SIWF informiert über die finanzielle Situation des SIWF. Das e-Logbuch 2.0 stellt mit einer jährlichen Investition von CHF 1 Mio. den grössten Budgetposten dar. Die Projektkosten werden eingehalten, jedoch gibt es insbesondere bei der Einführung des neuen Cockpits eine Verzögerung. Das Cockpit wird voraussichtlich im ersten Quartal 2019 zur Verfügung stehen. Für das Budget 2019 ist ein Gewinn von CHF 2000 vorgesehen. Massgeblich zu diesem ausgeglichenen Budget tragen die Zunahme der erteilten Facharzttitel wie auch verschiedene Gebührenerhöhungen bei. Auch die Weiterbildungsstätten sollen sich über die Zertifizierungs- und Visitationsgebühren vermehrt an den Aufwendungen des SIWF beteiligen. Bei den Facharzttiteln wird für den zweiten und alle weiteren eine Gebühr von CHF 2000 fällig. Die Gebühr für den ersten Facharzttitel bleibt unverändert bei CHF 4000 (seit 2002).

Aufwandseitig wird die Projektförderung wieder durchgeführt. Dafür werden CHF 150000 für innovative Projekte im Bereich der Weiterbildung eingesetzt. Sowohl bei den e-Projekten wie auch beim Personal ist 2019 mit einem Mehraufwand zu rechnen. Das Vermögen wird per 1.1.2020 voraussichtlich CHF 2,2 Mio. betragen.

Gemäss Finanzplanung 2020-2021 rechnet das SIWF mit steigenden Gewinnzahlen und das Vermögen sollte sukzessive wieder über CHF 3 Mio. steigen.

\subsection{Budget 2019 FMH}

Reto Heiz/Leiter a.i. der Abteilung Finanzen und Verwaltung erläutert im Detail das Budget 2019 der FMH, welches ein Defizit von CHF 144000 aufweist. Die von der ÄK genehmigte Einführung der Ausgabenbremse ab Budget 2016 wurde eingehalten und wird auch weiterhin fortgeführt. Positiv wirken sich auch die bis heute umgesetzten Massnahmen der Budgetstabilisierung auf das Budget 2019 aus.

Der minimale Verlust resultiert unter anderem aus erhöhten IT-Beratungskosten und Software-Entwicklungen. Der Personalaufwand bleibt unverändert, die Löhne Dritter und Gutachter fallen jedoch tiefer aus. Hingegen werden die Kosten für strukturelle Projekte, wie I-Governance, Gesamtsanierung Elfenstrasse und Monitoring TARCO in den kommenden Jahren zunehmen, was die finanzielle Planungssicherheit tangiert. Die zunehmenden Mitgliederzahlen wie auch die Reduktion der von der ÄK beschlossenen Sonderbeiträge vermögen die Aufwendungen knapp nicht zu decken.

Mit dem Budget 2019 wird belegt, dass die FMH auf der Grundlage der mittelfristigen Finanzplanung eine Disziplinierung bei den Ausgaben erlangt. 


\subsection{Budget 2019 GPK}

Reto Heiz/Leiter a.i. der Abteilung Finanzen und Verwaltung präsentiert das Budget der GPK, welches mit CHF 75000 bescheiden ausfällt. Die GPK ihrerseits trägt ebenfalls dazu bei, die verabschiedeten Budgetstabilisierungsmassnahmen umzusetzen.

\section{Bericht der GPK}

Dr. med. Adrian Sury/Präsident GPK rapportiert über die Sitzungstätigkeit im vergangenen Jahr, welche u.a. der Gesamtsanierung der Elfenstrasse wie auch der finanziellen Situation der FMH gewidmet war. Die GPK hat von der Detailplanung des Umbaus Kenntnis erhalten und ist beunruhigt wegen des Ausmasses der Kosten. Sie hat dem ZV bereits angeregt, die Delegierten der ÄK nochmals über dieses Sanierungsbudget abstimmen zu lassen. Die GPK hat sich intensiv mit dem Budget der FMH befasst und ist über die Kehrtwende beim SIWF erfreut. Das konsolidierte Budget der FMH weist einen Verlust von CHF 144000 aus. Der Lohnaufwand ist um CHF 1854000 höher budgetiert als beim Rechnungsabschluss 2017. Dies ist auf die neuen Anstellungen beim SIWF und im Generalsekretariat zurückzuführen. Die Entwicklung ist aufgrund der aktuell hohen Personalfluktuation schwierig abzuschätzen. Die Rückstellungen entsprechen den Notwendigkeiten und die Liquidität ist sehr gut. Die Anlagerenditen sind nach dem ausgezeichneten Resultat von 2017 schlechter. Aber durch die Neuausrichtung der Anlagestrategie mit weniger Obligationen und einer besseren Steuerung der Aktienanlagen konnte ein noch schlechteres Resultat verhindert werden.

Erfreulich ist ebenfalls die positive Auswirkung der bis heute umgesetzten Massnahmen im Rahmen der Budgetstabilisierung. Die GPK als Aufsichtsorgan der FMH empfiehlt den Delegierten der ÄK, das Budget der FMH und des SIWF anzunehmen. Adrian Sury/Präsident GPK dankt dem ZV, dem Generalsekretariat, dem Vorstand und der Geschäftsleitung des SIWF für die transparente Zusammenarbeit sowie die ausgezeichnete Gesprächskultur.

Jürg Schlup/Präsident FMH hält bezüglich der Gesamtsanierung der Liegenschaft an der Elfenstrasse fest, dass die Bedenken der GPK zurzeit unbegründet seien. Das für dieses Projekt verantwortliche ZV-Mitglied, Christoph Bosshard, wird heute über den aktuellen Stand der Gesamtsanierung und das weitere Vorgehen informieren. An der ÄK vom 9. Mai 2019 werden die Delegierten über die Detailplanung und die geplanten Gesamtkosten durch die Bauherrenschaft im Detail orientiert.

\section{Sonderbeitrag NAKO (Nationale Konsolidie-} rungsstelle)

Gemäss Urs Stoffel/ZV FMH und Departementsverant- wortlicher Ambulante Versorgung und Tarife ist die Datensammlung der praktizierenden Ärzte unerlässlich und wird immer wichtiger, um die Datenparität mit den Tarifpartnern zu sichern. Für die Weiterführung der Tarifrevision mit dem Projekt TARCO Etappe 2 wie auch für das vom BAG verlangte Monitoring ist die FMH auf diese Daten angewiesen. Der Sonderbeitrag dient zur Basisfinanzierung der Infrastruktur und zur Sicherstellung des Betriebs der NewIndex AG und ermöglicht damit der Ärzteschaft einen laufenden $\mathrm{Zu}$ gang zum NAKO-System als Grundlage zur gezielten Datenanalyse und Datenauswertung.

\section{Antrag ZV:}

Die ÄK genehmigt den Sonderbeitrag für 2019 zugunsten NAKO von CHF 40 für die Kategorien 1 und 2.

Beschluss:

Der Antrag wird mit 139 Ja, 7 Nein und 14 Enthaltungen angenommen.

\section{Sonderbeitrag Revue Médicale Suisse}

Reto Heiz/Leiter a.i. der Abteilung Finanzen und Verwaltung beantragt, dem Sonderbeitrag für 2019 zugunsten der Revue Médicale Suisse von CHF 10 zuzustimmen.

\section{Antrag ZV:}

Die ÄK genehmigt den Sonderbeitrag für 2019 zugunsten Revue Médicale Suisse von CHF 10 für die Kategorien 1, 2, 3, 5 und 6.

\section{Beschluss:}

Der Antrag wird mit 147 Ja, 3 Nein und 8 Enthaltungen angenommen.

\section{Sonderbeitrag SGAIM}

Dr. med. Philippe Luchsinger/SGAIM legt die Beweggründe für den am 4. Oktober 2018 eingereichten Antrag für einen einmaligen Unterstützungsbeitrag für die Initiative "Ja zum Schutz der Kinder und Jugendlichen vor Tabakwerbung» von CHF 5 dar. Das Ziel, innert der Initiativfrist raschmöglichst viele Unterschriften einzuholen, wurde verfehlt. Da es sich hier um eine auch für die FMH wichtige Initiative handelt, sind nach ihm alle Massnahmen zu ergreifen, um die noch nötigen Unterschriften einzuholen. In der jetzigen Konstellation wird das Tabak-Produktegesetz zu einem - gerade im Hinblick auf die Prävention zahnlosen Instrument und öffnet der Tabaklobby für ihre Arbeit Tür und Tor. Das gilt es zu verhindern. Er bedauert die fehlende Mobilisation innerhalb der Ärzteschaft, ist jedoch überzeugt, mit professioneller Unterstützung die ausstehenden Unterschriften zu sammeln. Ein einmaliger Sonderbeitrag würde ein positives Zeichen setzen. 
Antrag SGAIM:

Erheben des Sonderbeitrags für 2019 «Ja zum Schutz von Kindern und Jugendlichen vor Tabakwerbung» von CHF 5 für die Kategorien 1, 2, 3, 4, 5, 6, 7 und 11. Beschluss:

Der Antrag wird mit 90 Nein, 70 Ja und 6 Enthaltungen abgelehnt.

\section{Antrag VSAO zur Finanzierung von politischen Vorstössen}

Dr. med. Anja Zyska Cherix/Präsidentin VSAO betont, dass ihr Verband den Antrag der SGAIM wie auch die Volksinitiative "Ja zum Schutz von Kindern und Jugendlichen vor Tabakwerbung» unterstützt. Die Verbandsdelegieren bekunden jedoch Mühe mit dem von der SGAIM gewählten Vorgehen. Es stellen sich dabei grundsätzliche Fragen. Wie genau wollen die Initianten die zusätzlichen Mittel einsetzen? Handelt es sich um einen Einzelfall oder wird er Schule machen? Mit Blick auf die zweite Frage beantragt der VSAO, das Vorgehen bei Finanzbegehren im Zusammenhang mit politischen Vorstössen zu überdenken und eine generelle Lösung für alle zu finden. Dadurch sollten sich Anträge wie jener der SGAIM in Zukunft erübrigen.

\subsection{Mitgliederbeiträge}

Reto Heiz/Leiter a.i. der Abteilung Finanzen und Verwaltung präsentiert die Mitgliederbeiträge für 2019. Die Grundbeiträge bleiben zum Vorjahr unverändert.

Die ÄK genehmigt folgende Mitgliederbeiträge für 2019: Kategorien 1 und 2: $\quad$ CHF 760

Kategorie 3: $\quad$ CHF 485

Kategorie 4: $\quad$ CHF 355

Kategorie 5: $\quad$ CHF 294

Kategorie 6: $\quad$ CHF 188

Kategorie 7: $\quad$ CHF 142

Kategorie 11: $\quad$ CHF 178

Antrag ZV:

Genehmigung der Mitgliederbeiträge 2019.

Beschluss:

Die Mitgliederbeiträge 2019 werden mit 162 Ja, 0 Nein und 2 Enthaltungen genehmigt.

\subsection{Budget 2019 konsolidiert}

Antrag ZV:

Die ÄK verabschiedet das konsolidierte Budget 2019 der FMH mit einem Verlust von TCHF 144 (Budget 2019 des SIWF mit einem Gewinn von TCHF 2, Budget 2019 der FMH mit einem Verlust von TCHF 146).

Beschluss:

Das konsolidierte Budget der FMH 2019 wird mit $163 \mathrm{Ja}, 0$ Nein und 5 Enthaltungen angenommen.
Antrag VSAO

Antrag VSAO:

Der ZV der FMH legt der ÄK im Frühling 2019 einen Vorschlag vor, wie Anträge auf finanzielle Unterstützung von politischen Vorstössen über bestehende Fonds/Kassen der FMH finanziert werden. Beschluss:

Der Antrag wird mit 119 Ja, 37 Nein und 6 Enthaltungen angenommen.

\section{Bericht 2018 Budgetstabilisierungs- massnahmen}

Reto Heiz/Leiter a.i. der Abteilung Finanzen und Verwaltung gibt einen Gesamtüberblick über das im 1. Semester 2018 erreichte Ergebnis. Die von der ÄK am 30. Oktober 2014 festgelegten Ziele beinhalteten das Erreichen eines finanziellen Gleichgewichts zwischen den getätigten Ausgaben und den generierten Einnahmen. Die FMH soll sich in Zukunft auf ihre Kernaufgaben konzentrieren und Nebenaufgaben abbauen. Nur mit einer ausgewogenen Finanzlage ist gewährleistet, dass die FMH auch weiterhin als bedeutende Partnerin im Gesundheitswesen wahrgenommen wird und sich auf die Kernaufgaben konzentrieren kann. Die Einsparvorgaben von total CHF 5,8 Mio. sollten in zwei Etappen zwischen 2017 und 2019 erreicht werden; einerseits durch Aufwandverminderungen von CHF 3,8 Mio. und anderseits durch Ertragssteigerungen von CHF 2 Mio. Per Ende Dezember 2017 wurden 83,8\% realisiert.

Nach dem ersten Semester 2018 resultiert ein Defizit von TCHF 366. Das ist weniger als ursprünglich geplant. Die Hauptdifferenzen liegen sowohl bei Projektverzögerungen wie auch bei nicht vollständig umgesetzten Massnahmen im Bereich der Organe. Da es sich hier um einen Zwischenbericht handelt und sich verschiedene Resultate erst auf Ende des Geschäftsjahres 2018 präzise beziffern lassen, wird der Jahresabschluss 2018 aussagekräftiger ausfallen. Mit der von der ÄK 2014 genehmigten Ausgabenbremse will die FMH auch weiterhin sorgfältig mit den finanziellen Mitteln umgehen.

Die Delegierten nehmen den Stand der Umsetzungsarbeiten zu den Budgetstabilisierungsmassnahmen 1. Semester 2018 zur Kenntnis.

\section{Wahlen und Bestätigungswahlen}

\subsection{Bestätigungswahl von nominierten DV-Delegierten}

Art. 36a der FMH-Statuten sieht vor, dass die Delegierten und Ersatzdelegierten der DV durch die ÄK bestätigt werden. Wählbar sind ausschliesslich stimm- oder antragsberechtigte ÄK-Delegierte. 
Antrag:

Die antragsstellenden Organisationen VEDAG und KHM beantragen der ÄK, die folgenden Delegierten neu ab 25. Oktober 2018 für die DV der FMH zu bestätigen:

\section{VEDAG}

Bisher: Beat GAFNER, Niederscherli

Neu: Esther S. HILFIKER, Bern

KHM

Bisher: Marc MÜLLER, Grindelwald Franziska ZOGG-HARNISCHBERG, Zug

Neu: Heidi ZINGGELER FUHRER, Chur Philippe LUCHSINGER, Affoltern am Albis

Beschluss:

Die Delegierten werden mit 110 Ja, 3 Nein und 0 Enthaltungen bestätigt.

\subsection{Bestätigungswahl von nominierten DV-Ersatzdelegierten}

Art. 36a der FMH-Statuten sieht vor, dass die Delegierten und Ersatzdelegierten der DV durch die ÄK bestätigt werden. Wählbar sind ausschliesslich stimm- oder antragsberechtigte ÄK-Delegierte.

Antrag:

Die antragsstellenden Organisationen VEDAG, KHM und SFSM beantragen der ÄK, die folgenden Ersatzdelegierten neu ab 25. Oktober 2018 für die DV der FMH zu bestätigen:

VEDAG

Bisher: vakant

Neu: Lukas MEIER, Erlinsbach

KHM

Bisher: Philippe LUCHSINGER, Affoltern am Albis Neu: Franziska ZOGG-HARNISCHBERG, Zug

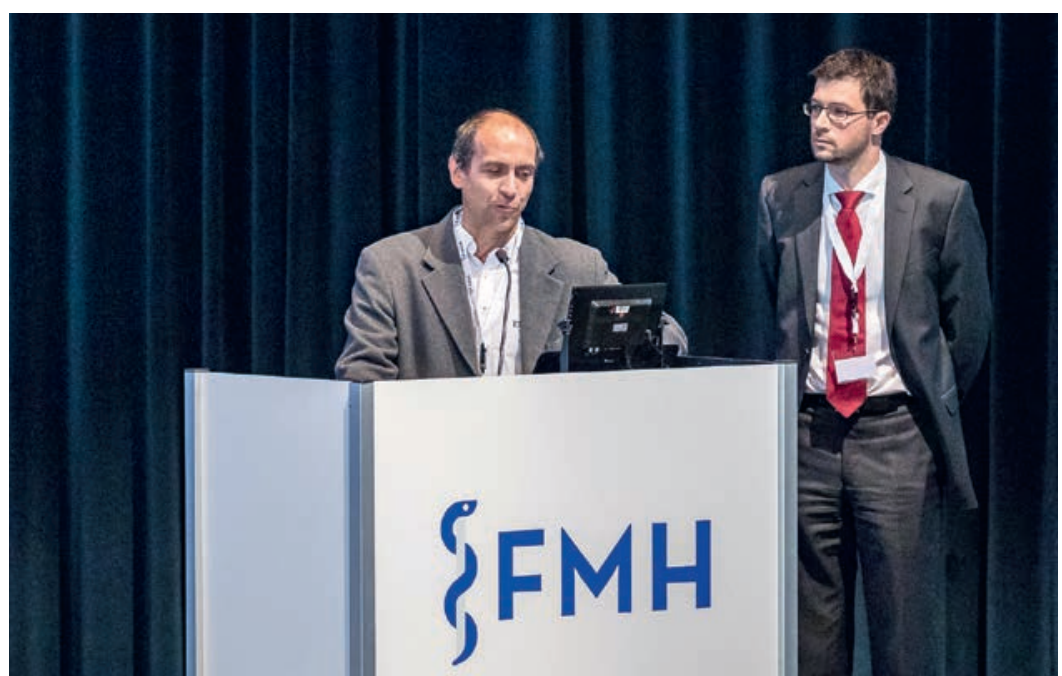

Dr. med. German Clénin (Mitte), Präsident der Schweizerischen Gesellschaft für Sportmedizin, hält ein Referat zum Thema «ärztliche Betreuung von Sporttreibenden». Rechts davon: Julien Duruz, Jurist der FMH.
SFSM

Bisher: vakant

Neu: Tobias EHMANN, Horw

Beschluss:

Die Ersatzdelegierten werden mit 110 Ja, 0 Nein und 3 Enthaltungen bestätigt.

\section{6. Änderung in Statuten, Geschäfts- ordnung und Standesordnung}

\section{1 Änderung in der Standesordnung (StaO)}

\subsection{1 Änderung Anhang 5: Richtlinien für die} ärztliche Betreuung von Sporttreibenden

Jürg Schlup/Präsident FMH begrüsst Dr. med. German Clénin/Präsident SGSM sowie RA Julien Duruz/RD FMH. $R A$ Julien Duruz weist auf die Neuerungen des Anhangs 5 hin. Die ÄK hat im April 2002 den Anhang 5 zur StaO verabschiedet. Dieser Anhang behandelt ein globales Thema, das die allgemeinen Grundsätze für die Betreuung von Sporttreibenden und die spezielle Frage des Dopings betrifft. Seit 2002 hat sich das Sportrecht erheblich weiterentwickelt, gerade in Bezug auf die Bekämpfung von Doping. Insbesondere ist 2012 ein neues Bundesgesetz über die Förderung von Sport und Bewegung (SpoFöG; SR 415.0) in Kraft getreten und die Sportvorschriften privater Verbände haben auf Anregung der Welt-Antidoping-Agentur (WADA) zahlreiche Weiterentwicklungen erfahren. Der Anhang 5 zur StaO FMH über die ärztliche Betreuung von Sporttreibenden ist überholt und muss überarbeitet werden, da er mittlerweile weder mit dem Bundesrecht noch mit den Vorschriften privater Verbände zur Dopingbekämpfung in Einklang steht. Der Revisionsentwurf wurde in Zusammenarbeit mit der SGSM und der Stiftung Antidoping Schweiz, dem Schweizer Kompetenzzentrum bei der Dopingbekämpfung, erarbeitet und ebenfalls bei den in der ÄK angeschlossenen Organisationen in Vernehmlassung gegeben.

Die wichtigsten Änderungen betreffen im Wesentlichen den Kampf gegen Doping. Bei den sportmedizinischen Grundsätzen wurden Anpassungen vor allem in formeller Hinsicht vorgenommen. Der Anhang soll aus standesethischer Perspektive einen globalen Ansatz in Dopingfragen entwickeln und alle Formen von Doping durch die Ärzteschaft verbieten. Die StaO würde damit in bestimmten Bereichen über das Bundes- und Sportrecht hinausgehen. So ist beispielsweise eine Ärztin oder ein Arzt, die oder der sich bei Teilnahme an einem Wettbewerb dopt, gesetzlich nicht strafbar, könnte aber wegen Verletzung der StaO und ihres Anhangs 5 sanktioniert werden. Es lässt sich aus berufsethischer Sicht auch nicht rechtfertigen, dass Ärztinnen und 
Ärzte ihr medizinisches Wissen einsetzen, um einen Wettbewerb, bei dem sie teilnehmen, zu verzerren.

Anhang 5 wird in seiner revidierten Fassung in Fragen der Dopingbekämpfung nicht nur eine solide Informationsquelle für die Ärzteschaft und die Standesorgane darstellen, sondern könnte auch als Instrument für Straf- und Zivilgerichte sowie Aufsichtsbehörden dienen, wenn es um die Definition/Auslegung beruflicher Pflichten im Zusammenhang mit Doping geht.

German Clénin/Präsident SGSM bringt die Sicht der SGSM ein und erläutert anhand von drei Fallbeispielen, welche Abklärungen durch Ärztinnen und Ärzte zu treffen sind, bevor Medikamente verabreicht werden. Die SGSM wurde bereits früh in die Überarbeitung des Anhangs 5 der StaO einbezogen. Der Vorstand der SGSM befürwortet die Annahme der aktuell vorliegenden überarbeiteten Version des Anhangs 5 der StaO "Ärztliche Betreuung von Sporttreibenden".

Für weiterführende Informationen dienen die untenstehenden Links:

- SGSM: https://sgsm.ch/

- Stiftung Antidoping Schweiz: https://www.antidoping.ch/

- Medikamentenabfrage: https://www.antidoping.ch/ de/medizin-substanzen-und-methoden/ medikamente-schweiz-abfrage

Nach den Diskussionen im Plenum dankt der Vorsitzende den beiden Referenten für ihre Präsentation und die geleistete Arbeit. Er verabschiedet German Clénin. Da es sich um zwei Anträge handelt, sind die Delegierten einverstanden, über diese zusammen abzustimmen.

Antrag ZV:

Die ÄK stimmt:

a. der beigefügten Anpassung von Art. 33bis der FMH-Standesordnung zu;

b. der beigefügten Revision von Anhang 5 der FMH-Standesordnung über die ärztliche Betreuung von Sporttreibenden zu.

\section{Beschluss:}

Die Anträge werden mit $152 \mathrm{Ja}, 3$ Nein und 3 Enthaltungen angenommen.

\subsection{2 Änderung SAMW-Richtlinie «Umgang mit Sterben und Tod»}

Jürg Schlup/Präsident FMH hält einleitend fest, dass der ZV die neue Richtlinie begrüsst und sich die Ablehnung des ZV einzig auf das Unterkapitel 6.2.1 bezieht. Der Begriff des «unerträglichen Leidens» ist ein undefinierter Rechtsbegriff, stellt die behandelnden Ärztinnen und Ärzte vor Probleme und führt zu Rechtsunsicherheit. Der ZV ist durchaus für eine Ausweitung der heutigen Regelung, aber auf einer sicheren juristischen
Grundlage. Er hat dazu auch einen Vorschlag formuliert und im März und Juli 2018 diesbezüglich Gespräche mit der SAMW geführt.

Der Präsident begrüsst Prof. Dr. med. Christian Kind, welcher als Vorsitzender der Subkommission der Zentralen Ethikkommission diese Richtlinie der SAMW erarbeitet hat. Die SAMW hat ihre Richtlinie «Betreuung von Patientinnen und Patienten am Lebensende» einer Totalrevision unterzogen und den neuen Entwurf mit dem Titel «Umgang mit Sterben und Tod» in die Vernehmlassung gegeben. Die SAMW hat die Richtlinie an ihrer Mai-Sitzung 2018 verabschiedet.

Prof. Kind stellt klar, dass in der neuen Richtlinie die Suizidhilfe ${ }^{1}$ keinesfalls das zentrale Thema ist. Im Gegensatz zur Vorgängerversion, die sich auf das unmittelbare Lebensende beschränkte, greift sie weiter. Sie gibt Hilfestellungen zum frühzeitigen Sprechen über Sterben und Tod bei Diagnose einer wahrscheinlich zum Tod führenden Krankheit. Sie regelt die Zulässigkeit und das Vorgehen für die palliative Sedierung und für den Umgang mit Patienten, die durch freiwilligen Verzicht auf Nahrung und Flüssigkeit aus dem Leben scheiden möchten. Zudem wird neu auch die Suizidhilfe für Patienten geregelt, bei denen der Tod nicht in den nächsten Tagen bis Wochen absehbar ist. In der neuen Richtlinie steht das Gespräch über Sterben und Tod im Zentrum und ist vom Leitgedanken der Palliative Care geprägt. Er verweist nochmals auf die Bedingungen für die Zulässigkeit der Suizidhilfe. Die Urteilsfähigkeit muss sorgfältig abgeklärt sein. Bei Vorliegen von Zuständen, welche diese häufig beeinträchtigen, wie z.B. einer psychischen Erkrankung oder einer Demenz, muss die Abklärung der Urteilsfähigkeit durch einen entsprechenden Facharzt vorgenommen werden. Der Wunsch des Patienten nach Suizidhilfe muss wohlerwogen, ohne äusseren Druck entstanden und dauerhaft sein. Diese Voraussetzungen müssen durch eine unabhängige Drittperson überprüft worden sein. Von den Kritikern der neuen Richtlinie wird verlangt, das Kriterium des «unerträglichen Leidens» zu streichen und durch die Bedingung zu ersetzen, dass eine tödliche, nicht behandelbare Krankheit vorliegen müsse. Dies ist für die SAMW kein geeigneter Weg zur Regelung der Suizidhilfe. Der Begriff «tödliche Krankheit» ist zwar objektiv, lässt sich aber kaum klar abgrenzen. Weiter werde von den Gegnern kritisiert, dass die Richtlinie die emphatische Orientierung ärztlichen Handelns am Patientenwohl zugunsten einer absoluten Respektierung der Selbstbestimmung aufgeben würde. Dies trifft nicht zu. Die Selbstbestimmung des urteilsfähigen Patienten muss zwingend respektiert werden. Der Arzt darf nichts tun, was dem Patientenwohl abträglich ist, selbst wenn der Patient dies ver-
Synonym wird auch Begriff «ärztliche Suizidbeihilfe» verwendet 
langt. Das grosse Problem bei der Suizidhilfe ist, dass die Meinungen darüber, ob diese grundsätzlich dem Wohl des Patienten dienen könne oder nicht, unter Ärzten und Ethikern, wie auch in der breiten Öffentlichkeit der Schweiz geteilt sind. Deshalb hat die SAMW beschlossen, dieser Realität Rechnung zu tragen, indem sie die Suizidhilfe als kontrovers diskutierte medizinische Handlung der Entscheidung des einzelnen Arztes nach bestem Wissen und Gewissen überantwortet. Die Suizidhilfe gilt aber nicht als allgemein akzeptierte medizinische Handlung, und darf deshalb vom Arzt nicht von sich aus dem Patienten angeboten werden. Das Verlangen danach muss vom urteilsfähigen $\mathrm{Pa}$ tienten ausgehen. Auf den Sterbewunsch soll emphatisch eingegangen und medizinisch-therapeutische Optionen sowie andere Hilfs- und Unterstützungsmöglichkeiten zur Linderung des Leidens müssen gesucht und angeboten werden. Erst wenn dies erfolglos geblieben ist, darf auf das Verlangen nach Suizidhilfe eingetreten werden. Schliesslich wird von den Gegnern auch bemängelt, dass die neue Richtlinie die Suizidprävention gefährden würde.

Zusammenfassend lässt sich sagen, dass die Richtlinie den behandelnden Ärzten die Freiheit gibt, nach ihrer persönlichen ethischen Haltung zu handeln. Wer sich grundsätzlich nicht vorstellen kann, Suizidhilfe zu leisten, muss dies nicht tun. Er soll aber dem Patienten mitteilen, dass er nicht dazu verpflichtet werden kann. Wenn ein Arzt also grundsätzlich in Einzelfällen zur Suizidhilfe bereit ist, aber bei einem bestimmten $\mathrm{Pa}$ tienten dessen Leiden nicht nachvollziehen kann, muss er das Begehren abweisen. Wenn er dagegen in einem konkreten Fall überprüft hat, dass alle Bedingungen erfüllt sind und er überzeugt ist, zum Wohl des Patienten zu handeln, dann ist er durch die Richtlinie gestützt. In diesen Fällen gibt die Richtlinie mehr Sicherheit. Für die übrigen ändert sich bezüglich Suizidhilfe nichts. Dass eine Ablehnung durch die ÄK den Gesetzgeber dazu motivieren könnte, die Suizidhilfe auf gesetzlicher Ebene neu zu regeln, hält Prof. Kind für wenig aussichtsreich, sind doch alle bisherigen Bemühungen gescheitert. Aus ethischer Sicht erscheint eine detaillierte gesetzliche Regelung eines so komplexen und in wesentlichen Stücken privaten Problems auch nicht wünschbar. Dass eine Anwendung der neuen Richtlinie die Häufigkeit der Suizidhilfe massgeblich beeinflussen könnte, erscheint ihm ebenfalls wenig wahrscheinlich. Wer in den letzten Jahren in der Schweiz unbedingt Suizidhilfe in Anspruch nehmen wollte, hat sie in der Regel auch bekommen.

Eine Beibehaltung der alten SAMW-Richtlinie in der StaO würde bedeuten, dass zwei unterschiedliche Richtlinien zur gleichen Zeit bestehen, was insbeson- dere für Ärzte, die grundsätzlich bereit sind, Suizidhilfe zu leisten, zu einer neuen Rechtsunsicherheit führen würde.

Jürg Schlup/Präsident FMH hält fest, dass bei Ablehnung der neuen SAMW-Richtlinie die alte Richtlinie, welche bereits heute Teil der StaO der FMH ist, weiterhin ihre Gültigkeit behält. Nach den Verständnisfragen verabschiedet der Präsident Prof. Kind und dankt ihm bestens für seine Ausführungen.

Die anschliessende Diskussion eröffnet Dr. Michael Barnikol/Jurist Rechtsdienst FMH. Er unterstreicht, dass sich - nach seinem Dafürhalten - zwar viele Ärzteorganisationen im Rahmen des Vernehmlassungsverfahrens geäussert haben, diese aber eher zur Richtlinie insgesamt Stellung bezogen haben, jedoch häufig nicht zur Frage der Suizidhilfe (Unterkapitel 6.2.1). Die Aussage, wonach die weit überwiegende Mehrheit der Ärztegesellschaften sich explizit für die Suizidhilferegelung in der neuen Richtlinie der SAMW ausgesprochen haben, ist seiner Meinung nach nicht ganz korrekt.

Er geht auf die verschiedenen Fragen und inhaltlichen Punkte ein, die sich im Zusammenhang mit dieser Revision stellen. Nach Genehmigung durch die ÄK würde diese Regelung Teil der StaO der FMH und damit in standesrechtlicher Hinsicht verbindlich. Im Falle eines Verstosses könnten die kantonalen Standeskommissionen Sanktionen aussprechen. Die Anwendbarkeit der Suizidhilfe in Ziff. 6.2.1 der neuen Richtlinie geht erheblich weiter, als die alte Regelung. Der Begriff «unerträgliches Leiden» ist sehr unbestimmt und für den betroffenen Arzt schwer überprüfbar, was für die Ärztinnen und Ärzte einen erheblichen Unsicherheitsfaktor darstellt. Die Ärzteschaft hat ein Interesse an klar formulierten Kriterien. Die SAMW wollte mit der neuen Richtlinie der gesellschaftlichen Realität gerecht werden, dass Patienten nicht nur am Lebensende den Wunsch nach Suizidhilfe äussern. Aber juristisch betrachtet, ist die vorgeschlagene Regelung unausgewogen.

Philippe Luchsinger/SGAIM erläutert, dass für die SGAIM die Richtlinien klar sind und bei Ablehnung des DVAntrags die neuen SAMW-Richtlinien in die StaO aufzunehmen seien. So unterschiedlich Menschen gelebt haben, so unterschiedlich wollen sie auch sterben. Der Patient muss urteilsfähig sein. Der Wunsch ist ohne Druck persistent und begründet auf einem Leiden, an der der Patient unerträglich leidet und bei dem andere Behandlungsoptionen erfolglos geblieben sind. Und wenn möglich, soll die Umgebung des Patienten in diesen Prozess einbezogen werden. Es wird immer Unsicherheiten im Alltag geben. Jeder Arzt soll die Möglichkeit haben, nach den eigenen Vorstellungen und nicht aufoktroyiert durch Haltungen anderer 
seine wichtigste Tätigkeit wahrnehmen zu können, nämlich die Patientinnen und Patienten in allen Lebenssituationen - und zu denen gehört auch das Sterben - zu begleiten, zu betreuen und ihnen beizustehen, auch im Fall eines assistierten Suizids.

Pierre Vallon/Präsident DV zeigt nochmals die Beweggründe der DV auf, die zu ihrem Antrag führten. In seiner Stellungnahme hat der ZV das Kriterium, wonach eine Suizidhilfe nicht mehr am Lebensende, sondern bereits beim Vorliegen eines «unerträglichen Leidens» des Patienten zulässig sein soll, als zu unbestimmt kritisiert. Der ZV schlägt vor, die Zulässigkeit der Suizidhilfe auf solche Patienten zu beschränken, die an einer schweren, tödlichen Krankheit leiden und deren $\mathrm{Zu}$ stand sich bei einer sachgerechten medizinischen Behandlung auch nicht bessern wird. Die DV hat lange über die Aufnahme dieser zur Diskussion stehenden Richtlinie in die StaO debattiert und sich schlussendlich gegen die Aufnahme entschieden. Diese Ablehnung wurde damit begründet, dass die Ärzteschaft alleine die Verantwortlichkeit für die ärztlich assistierte Suizidhilfe trage und in grosser Sorge bezüglich der gesellschaftlich und juristisch korrekten Durchführbarkeit der vorliegenden Bestimmung sei. Die DV will die neue Richtlinie erst dann in die StaO übernehmen, wenn der Begriff «unerträgliches Leiden» durch einen klar definierten Rechtsbegriff von der SAMW ersetzt wird.

Nach dem Abarbeiten einer langen Rednerliste, stellt der Präsident einen Ordnungsantrag.

Antrag ZV:

Die Rednerliste wird nach den drei nächsten Rednern abgeschlossen.

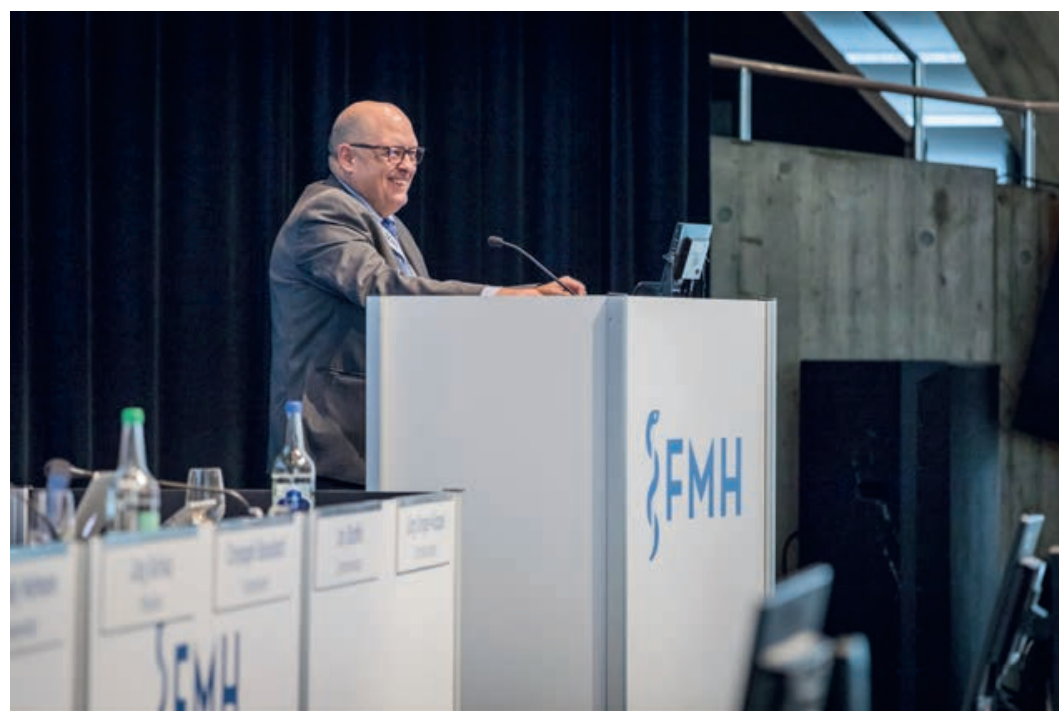

Der DV-Präsident, Pierre Vallon, präsentiert den von der Delegiertenversammlung eingereichten Antrag.

\section{Beschluss:}

Der Ordnungsantrag wird mit grossem Mehr gutgeheissen.

Nach der intensiv geführten Debatte zeigt sich für eine Mehrheit, dass die Aufnahme der neuen SAMW-Richtlinie $\mathrm{zu}$ einer grossen Rechtsunsicherheit führen würde. Die FMH möchte in ihrer StaO klare Richtlinien für Ärztinnen und Ärzte sowie auch für Patientinnen und Patienten vorgeben. Diese sollen sich nach objektiv überprüfbaren Kriterien richten.

Die FMH könnte sich durchaus eine weitere Liberalisierung vorstellen und schlägt vor, dass die geregelte Suizidhilfe sich auf solche Patienten beschränken sollte, die an einer schwerwiegenden tödlichen Krankheit leiden und deren Zustand sich bei einer sachgerechten medizinischen Behandlung auch nicht bessern wird. Eine entsprechende Diagnose kann ein Arzt mit hinreichender Zuverlässigkeit stellen.

Jürg Schlup/Präsident FMH stellt den Antrag, die zwei Anträge gegenüberzustellen.

\section{Antrag ZV:}

Der Antrag der DV wird dem Antrag der SGAIM gegenübergestellt.

Beschluss:

Der Antrag wird mit grossem Mehr, 6 Nein und 5 Enthaltungen angenommen.

\section{Antrag DV:}

Die ÄK beschliesst, dass die Richtlinie der SAMW «Umgang mit Sterben und Tod» nicht in die StaO der FMH übernommen werden, bis der Begriff des "unerträglichen Leidens" in Kapitel 6.2.1 der Richtlinie durch klar definierte Rechtsbegriffe ersetzt wird.

Antrag SGAIM:

Die neue Richtlinie 2018 der SAMW «Umgang mit Sterben und Tod» ist in die SaO aufzunehmen.

Beschluss:

Für den Antrag der DV stimmen 99 Delegierte; für den Antrag der SGAIM stimmen 37 Delegierte. 5 Delegierte enthalten sich der Stimme.

Nach Annahme des Antrags der DV wird die neue Richtlinie der SAMW «Umgang mit Sterben und Tod» nicht in die StaO der FMH übernommen. Die Richtlinien von 2012 «Betreuung von Patientinnen und Patienten am Lebensende» behalten für die Mitglieder der FMH weiterhin Gültigkeit, auch wenn sie im Juni 2018 von der SAMW zurückgezogen wurden.

Der ablehnende Entscheid wird der SAMW mitgeteilt. Sobald ein neuer Vorschlag seitens der SAMW vorliegt, wird dieser wiederum der ÄK vorgelegt. 


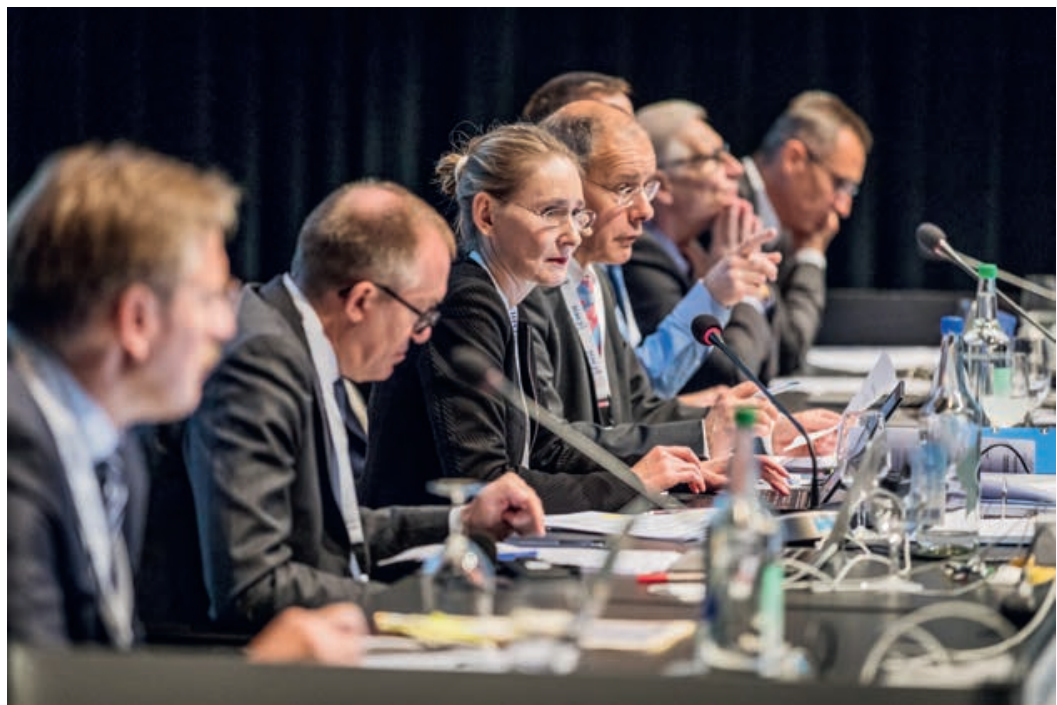

Seit 1. Oktober Generalsekretärin der FMH: Dr. iur. Ursina Pally Hofmann.

6.1.3 Antrag zur Revision der Standesordnung (Art. 23, Art. 30, Anhang 2 und Anhang 3)

Dr. med. Josef Brandenberg/Präsident FMCH führt aus, dass viele Mitglieder der FMCH auf die mfe-Kampagne empört reagierten haben. Entsprechende Massnahmen, welche von einer Gegenkampagne bis zu einem Austritt aus den Gremien oder sogar aus der FMH reichten, wurden gefordert. Die Kampagne gefährdet die Einheit des Berufsverbandes in einer heiklen Phase. Die StaO der FMH erlaubt es heute, gegen Mitglieder vorzugehen, jedoch nicht gegen eine Fachgesellschaft. Die FMCH verlangt eine Revision der StaO, um künftig auch gegen Ärzteorganisationen vorgehen zu können.

Philippe Luchsinger/Präsident mfe und Kampagnen-Verantwortlicher betont, dass es nie die Absicht war, mit dieser Imagekampagne jemanden zu verletzen. Die Schweiz braucht mehr Haus- und Kinderärzte, weshalb die DV der mfe den entsprechenden Auftrag erteilte. Ziel war die Attraktivität der Hausarztmedizin ins Zentrum zu stellen. Die Kampagne richtete sich an Politiker, Medizinstudenten, an die Medien und Öffentlichkeit. Die mfe wählte ein unkonventionelles Vorgehen, um das Zielpublikum zu überraschen. Die Kernbotschaft war, dass 94\% der gesundheitlichen Probleme von einem Haus- oder Kinderarzt gelöst werden können. Diese Erkenntnis ist wissenschaftlich durch eine Studie des Instituts für Hausarztmedizin Zürich hinterlegt. Er bedauert es als Präsident der mfe, dass einzelne durch diese Kampagne verletzt wurden und entschuldigt sich dafür. Die mfe wird diese Videos auf dem Internet löschen.

Josef Brandenberg/Präsident FMCH dankt jenen mfeMitgliedern, die sich von dieser Aktion distanzieren.
Wenn die Verantwortlichen bei mfe die entsprechenden Löschungen auf dem Internet vornehmen, wird sich die Situation bei den Mitgliedern der FMCH beruhigen. Der Dachverband erachtet jedoch eine Klärung der Frage nach Sanktionen für Ärzteorganisationen als wichtig und verlangt die Revision der entsprechenden Artikel in der StaO der FMH.

Ursina Pally Hofmann/Generalsekretärin und Leiterin Rechtsdienst FMH weist auf die rechtlichen Aspekte hin. Die von der FMCH vorgeschlagenen Änderungen gleichen einem Systemwechsel mit langfristiger und umfassender Wirkung und wären nicht praktikabel. Die heutigen Regeln in der StaO gelten für Ärzte als natürliche Personen. Laut Statuten der FMH committen sich die Mitglieder, die Statuten wie auch die StaO einzuhalten. Anders verhält es sich bei den Ärzteorganisationen. Hier geht es nicht um berufstätige Ärzte, die Patienten behandeln, sondern um Interessenvertretung. Die Ärzteorganisationen der FMH committen sich denn auch nicht, die StaO, sondern nur die Statuten einzuhalten. Eine Änderung kann nur über eine Statutenrevision in die Wege geleitet werden. Dies bedingt jedoch eine gut durchdachte systematische Redaktion inklusive Änderung des Verfahrensrechts.

Nach einigen Wortmeldungen stellt Felix Kurth/VSAO den Ordnungsantrag, jetzt über den Antrag abzustimmen. Antrag VSAO:

Beendigung der Wortmeldung und Durchführung der Abstimmung.

Beschluss:

Der Antrag wird mit grossem Mehr gutgeheissen.

\section{Antrag FMCH:}

Die Standesordnung der FMH sei wie folgt zu ergänzen:

Art. 23 Kollegiales Verhalten, unzulässige Kritik Arzt und Ärztin (neu) sowie Ärzteorganisationen (Dachorganisationen, kantonale Ärztegesellschaften, Fachgesellschaften, ärztliche Berufsverbände) pflegen unter sich kollegiale Beziehungen, welche von Ehrlichkeit und Höflichkeit getragen sind. Jede Handlungsweise, die einen Kollegen oder eine Kollegin und in der persönlichen oder beruflichen Ehre ungerechtfertigterweise verletzt, ist zu unterlassen.

(Neu) Jede Handlungsweise, die Ärzteorganisationen, medizinische Fachgebiete, ärztliche Berufsgruppen und deren Mitglieder in der Ehre ungerechtfertigterweise verletzt, ist zu unterlassen.

Gegenüber Dritten bleiben Arzt und Ärztin in ihren Äusserungen über die Behandlungsweise eines Kollegen oder einer Kollegin sachlich und objektiv. 
(Neu) Gegenüber Dritten bleiben Ärzteorganisationen in ihren Äusserungen über die Tätigkeit anderer Ärzteorganisationen und deren Mitglieder sachlich und objektiv.

Art. 30 Beilegung von Streitigkeiten

Streitigkeiten unter Kollegen und Kolleginnen (neu) sowie unter Ärzteorganisationen, die auf einer Verletzung der Standesordnung, im Besonderen auf unkollegialem Verhalten beruhen, sollen direkt oder durch Vermittlung einer Drittperson bereinigt werden. Schlägt der Versuch der gütlichen Einigung fehl, ist der Streit vor dem für die Durchsetzung der Standesordnung zuständigen Organ auszutragen.

Anhang 2 zur Standesordnung der FMH enthält Richtlinien zu "Information und Werbung»

Punkt 2. Unzulässige Werbung (Art. 20 Abs. 2 StaO)

2.3. Die Information beeinträchtigt das Ansehen des Arztberufes insbesondere,

- wenn sie vergleichend Bezug nimmt auf Berufsangehörige wie z.B. herabsetzende Äusserungen über Kollegen und Kolleginnen, ihre Tätigkeit und deren medizinischen Methoden;

- (neu) wenn sie sich herabsetzend über die Tätigkeit anderer Ärzteorganisationen, Fachgebiete, Berufsgruppen und deren Mitglieder äussert.

Anhang 3 zur Standesordnung FMH Richtlinien für die Medientätigkeit von Ärztinnen und Ärzten (neu) sowie Ärzteorganisationen

Punkt 2. Die eigene ärztliche Leistung soll nicht betont, die Leistungen und Methoden anderer Ärzte und Ärztinnen (neu) sowie Ärzteorganisationen nicht abschätzig oder polemisch beurteilt werden.

Beschluss:

Der Antrag wird mit 105 Nein, 3 Ja und 10 Enthaltungen abgelehnt.

Pierre Vallon/Präsident $D V$ regt an, diese Thematik in der DV vertieft und mit genügend Zeit zu diskutieren. Seiner Meinung nach ist die DV das richtige Gremium dafür.

Jürg Schlup/Präsident FMH unterstützt den Vorschlag von Pierre Vallon/Präsident DV. Er empfiehlt der FMCH, die weiteren Schritte zu planen, aber unter Berücksichtigung der Einwände des Rechtsdienstes. Die FMH verfügt zum heutigen Zeitpunkt über keine rechtliche Grundlage, Massnahmen gegen angeschlossene Ärzteorganisationen zu ergreifen.

Für Josef Brandenberg/Präsident FMCH hängt das weitere Vorgehen davon ab, ob die mfe aufgrund der heutigen Voten die Videos auf dem Internet löschen wird.

\section{MAS - Strukturdaten Arztpraxen und ambulante Zentren}

Dr. med. Christoph Bosshard/Vizepräsident FMH und Departementsverantwortlicher Daten, Demographie und Qualität blickt einleitend auf die erste Datenerhebung zurück, welche im Juni 2017 abgeschlossen wurde. Diese diente rein statistischen Zwecken. Art. $30 \mathrm{KVV}$ Art. 59a KVG wie auch das Bearbeitungsreglement galten für diese Erhebung noch nicht. Die FMH hat zusammen mit dem Bundesamt für Statistik (BFS) anschliessend die Plausibilisierung der Daten durchgeführt. Das BFS hat die Auswertung am 10. April 2018 publiziert. Die Ergebnisse aus der Erhebung MAS 2015 sind für die FMH und die Ärzteschaft von zentraler Bedeutung und von Interesse. Der Aufbau der Statistiken der ambulanten Gesundheitsversorgung soll als Beitrag für ein integrales statistisches Gesundheitsinformationssystem dienen.

Die zweite Erhebung wird, basierend auf dem Datenjahr 2017, am 12. November 2018 starten. Diese Erfassung dient neben den statistischen neu auch den aufsichtsrechtlichen Zwecken. Erstmalig gilt die aufsichtsrechtliche Zweckbindung gemäss Art. 59 KVG und dem darauf basierenden Art. 30 der Verordnung sowie das Bearbeitungsreglement. Für Leistungserbringer, die an der Erhebung nicht teilnehmen, wird das BFS keine Sanktionen vorsehen. Die Vorjahresdaten aus der Erhebung 2015 können importiert werden.

Christoph Bosshard begrüsst Marco D'Angelo/Vizedirektor und Leiter Abteilung Gesundheit und Soziales BFS, der einige Resultate aus der ersten Erhebung präsentiert wie auch über die weiteren Schritte informiert. Die erste Erhebung wurde Mitte 2017 erfolgreich abgeschlossen. Im Rahmen dieser Erhebung wurden 18723 Unternehmen befragt. Rund drei Viertel der angeschriebenen Arztpraxen und ambulanten Zentren lieferten Angaben zu ihrer Situation. Fast 7000 Arztpraxen und ambulante Zentren, und damit die Hälfte der zur bereinigten Grundgesamtheit gehörenden Unternehmen, haben den Fragebogen ausgefüllt und Daten geliefert. Die ersten Ergebnisse wurden im April 2018 publiziert. Die zweite Erhebung wird in Erfüllung des gesetzlichen Auftrags von Art. 59a KVG zusätzlich auch zu aufsichtsrechtlichen Zwecken erhoben. Das für die Daten 2017 gültige Bearbeitungsreglement wurde am 20. März 2017 in Kraft gesetzt. Die für aufsichtsrecht liche und statistische Zwecke weitergegebenen Daten der Arztpraxen und ambulanten Zentren sind gemäss Bearbeitungsreglement anonymisiert. Die Arztpraxis wie auch der einzelne Arzt sind zu keinem Zeitpunkt wieder erkennbar. Das BFS hat für die zweite Erhebung 
den Fragenbogen optimiert und wiederum eine entsprechende Hotline eingerichtet.

Christoph Bosshard/Vizepräsident FMH und Departementsverantwortlicher Daten, Demographie und Qualität bezeichnet die Zusammenarbeit mit dem BFS als konstruktiv und zielgerichtet und dankt Marco D’Angelo für sein Kommen. Für weitere Informationen und Auskünfte steht das Departement für Daten, Demographie und Qualität den Mitgliedern gerne zur Verfügung.

\section{Antrag VSÄG: «Monistische Finanzierung"}

Die Walliser Ärztegesellschaft (VSÄG) hat am 20. September 2018 und die SMSR am 9. Oktober 2018 die entsprechenden Anträge eingereicht. Jürg Schlup/Präsident $F M H$ verweist in diesem Zusammenhang auf den Beschluss der DV vom 29. Juni 2017. Die DV hat damals einstimmig, ohne Gegenstimme und ohne Enthaltung, einer einheitlichen Finanzierung stationär und ambulant erbrachter medizinischer Leistungen zugestimmt. Sie hat dabei bevorzugt, dass das Geld der Kantone via eine gemeinsame Einrichtung KVG an die Versicherer geht.

Dr. med. Monique Lehky Hagen/Präsidentin VSÄG geht auf die Problematik der monistischen Finanzierung ein. Initial war eigentlich die Verschiebung der Kosten vom stationären auf den ambulanten Sektor geplant. Mittlerweise muss festgestellt werden, dass die Ziele der einheitlichen Finanzierung nicht erreicht werden. Dagegen zeichnet sich durch eine von verschiedenen Kantonen gewünschte Steuerung des gesamten ambulanten Gesundheitsbereichs das Risiko einer Aufhebung des Vertragszwangs ab. Es ist zu befürchten, dass eine solche Steuerung zu Ungunsten des nicht spitalambulanten Sektors ausfallen würde. Weiter wird diese Vorlage den politischen Druck auf alle ambulanten kantonalen Tarifverhandlungen erhöhen. Die vorgeschlagene KVG-Anpassung führt zu einem noch grösseren administrativen Aufwand. Ziel dieses Antrags war, auf diese Situation aufmerksam zu machen, damit die FMH raschmöglichst eine Kurskorrektur einleitet.

Dr. med. Philippe Eggimann/Präsident SMSR legt dar, dass seit dem Zeitpunkt, als die DV die Unterstützung der einheitlichen Finanzierung beschlossen hat, die Kantone ihre Vorschläge modifiziert haben. Die SMSR schlägt in ihrem ersten Antrag vor, dass die FMH ihre weitere Unterstützung für die monistische Finanzierung an die Bedingung knüpft, dass die Kantone auf die Kontrolle und verstärkten Planung spitalexterner ambulanter Tätigkeiten verzichten. Die aktuell vorge- schlagene monistische Finanzierung sieht vor, dass die Kantone mindestens 25,5\% der Kosten übernehmen, die nach Abzug der Selbstbehalte und der von den Versicherten geleisteten Anteile noch von den Versicherern zu tragen sind.

Der zweite Antrag der SMSR unterstützt eine differenzierte Finanzierung. Sie fordert, dass eine einheitliche Finanzierung nur für Leistungen gelten soll, die sich aus der Verlagerung von der stationären zur ambulanten Behandlung ergeben. Insbesondere soll das für Leistungen gelten, für die der Bund und/oder die Kantone eine Erstattung nur dann garantieren, wenn sie ambulant erbracht werden.

Dr. med. Josef Widler/Präsident AGZ stellt fest, dass sich die Situation zwischenzeitlich verändert hat. Seiner Meinung nach läuft die FMH mit den drei eingereichten Anträgen Gefahr, sich in ihrer Verhandlungsposition einzuschränken. Der heute von der AGZ eingereichte Antrag sieht vor, dass die FMH die monistische Finanzierung unterstützt. Sollte es bei der Umsetzung zu einer Steuerung der ambulanten Versorgung durch die Kantone kommen, ist es unabdingbar, dass die kantonalen Ärztegesellschaften partnerschaftlich und verbindlich in die Planung einbezogen werden. Josef Widler/Präsident AGZ bittet die Delegierten, den Antrag der AGZ zu unterstützen und fragt die beiden Antragsteller der VSÄG und der SMSR, ob sie ihre Anträge zurückziehen.

Pierre Vallon/Präsident DV erinnert nochmals an den Entscheid der DV vom 29. Juni 2017. Die heute aufgeworfenen Fragestellungen sind komplex und bedürfen einer intensiveren Diskussion. Er schlägt vor, dieses Thema an einer nächsten DV zu vertiefen.

Jürg Schlup/Präsident FMH unterstützt den Vorschlag von Pierre Vallon/Präsident DV. Die Uhr zeigt bereits 18.00 Uhr. Deswegen dürfen an dieser Sitzung gemäss heutigem Kammerentscheid keine Beschlüsse mehr gefasst werden. Er bittet die Antragsteller, ihre Anträge fristgerecht für die nächste DV einzureichen.

\section{Gesamtsanierung Bürogebäude Bern}

Christoph Bosshard/Vizepräsident FMH erinnert an den Beschluss der ÄK vom 26. Oktober 2017, an der sich die Delegierten für eine Gesamterneuerung mit Grundsanierung der Liegenschaft Elfen-strasse entschieden haben. Diesem Entscheid vorausgegangen war eine längere Debatte, welche sich auch über verschiedene Varianten von Verkauf und Neukauf, Vermietung und Verpachtung der Liegenschaft bis zur Grundsanierung und Weiternutzung erstreckte.

In Umsetzung des ÄK-Entscheides hat der ZV ein projektverantwortliches Gremium bestimmt, welchem 
die Aufgaben der weiteren Projektentwicklung übertragen wurde. Basierend auf der dem $\ddot{\mathrm{AK}}$ - Beschluss zugrundeliegenden Machbarkeitsanalyse hat der ZV vertiefte Abklärungen in den Bereichen Bausubstanz inkl. Risikoschadstoffe getätigt. Ebenfalls wurde zur Konkretisierung der Planung eine detaillierte Bedarfsanalyse durchgeführt, welche nicht nur das Gebäude, sondern die ganze Liegenschaft umfasste. Diese Analyse- und Planungsarbeiten wurden im Sommer abgeschlossen. Das daraus resultierende Vorprojekt wurde vom ZV am 22. August 2018 verabschiedet. Das Vorprojekt ist einerseits mit einer Plangenauigkeit von plus/minus 20\% behaftet, andererseits zeigt es aufgrund vorsichtigerer Schätzungen, dass allenfalls mit Kosten leicht oberhalb der verabschiedeten Bandbreite zu rechnen ist. Projektphasenbedingt wurde die Ersatzstandortfrage während der Umbauphase noch nicht konkretisiert. Der Markt zeigt sich jedoch relativ dynamisch und kundenfreundlich.

Die definitive Detailplanung der Sanierung wird der ZV im Frühjahr 2019 verabschieden. Anschliessend wird die Baueingabe vorbereitet. Ein Beginn der Umbauarbeiten ist erst ab November 2019 möglich. Die ÄK wird am 9. Mai 2019 über die weitere Projektentwicklung informiert und über begründete Anträge befinden, sofern der gesprochene Budget-Rahmen nicht eingehalten werden kann.

\section{Informationen aus Zentralvorstand, Generalsekretariat, SIWF und Abteilungen}

Die Delegierten haben die Informationen aus ZV, Generalsekretariat, SIWF und den Abteilungen vorgängig schriftlich erhalten.

Dr. med. Yvonne Gilli/ZV-Mitglied und Departementsverantwortliche Digitalisierung und eHealth gibt zusätzlich eine kurze mündliche Zusammenfassung über die Umsetzung von Massnahmen betreffend die europäische Datenschutz-Grundverordnung (DSGVO). Das DSGVO hat unter bestimmten Bedingungen Auswirkungen auf Spitäler und Praxen. Mit der verpflichtenden Umsetzung der DSGVO für europäische Mitgliedstaaten auf den 25. Mai 2018 sind Schweizer Unternehmen aufgrund der extraterritorialen Wirkung betroffen.

Der ZV hat der ÄK vom 26. Oktober 2017 beantragt, dass alle Organe der FMH, ihre Organisation und Prozesse im Hinblick auf die Erfüllung der Mindestanforderungen der Datenschutzgesetzrevision überprüfen und allfällig notwendige Korrekturmassnahmen zeitnah umsetzen. Die Delegierten sind auf diesen Antrag nicht eingetreten.

Seit Inkrafttreten der EU-DSGVO im Mai 2018 ist eine Welle von Fragen in diesem Zusammenhang an das zuständige Departement zu verzeichnen. Auch wenn nur wenige Arztpraxen und Spitäler von dieser neuen Verordnung direkt betroffen sind, hat das Departement Digitalisierung und eHealth ein Dokument «Fragen und Antworten» zusammengestellt, welches auf die Anwendbarkeit und die Auswirkung der Verordnung eingeht. Um das Risiko der Anwendbarkeit der DSGVO gering zu halten, hat das Departement zusätzlich eine Datenschutzerklärung in Zusammenarbeit mit der HIN AG erarbeitet. Die Datenschutzerklärung ist grundsätzlich für Ärztinnen und Ärzte, welche Informationen oder Dienstleistungen auf einer eigenen Webseite verfügbar machen, gedacht.

Weitere Informationen sind unter https://www.fmh.ch/ politik_themen/ehealth/datenschutz.html einsehbar.

\section{Varia}

Dr. med. Angelo Canova/AGZ weist auf die MehrfachMandate von Delegierten hin. Um eine pluralistische Meinungsbildung auch in Zukunft zu gewährleisten, schlägt er vor, dass Kandierende bei Wahlen ihre verschiedenen Ämter offenlegen.

\section{Termine ÄK 2019}

Donnerstag, 9. Mai 2019 (nicht wie ursprünglich festgelegt 2. Mai 2019)

Donnerstag, 31. Oktober 2019

\section{Termine ÄK 2020}

Donnerstag, 14. Mai 2020

Donnerstag, 29. Oktober 2020

Jürg Schlup/Präsident FMH schliesst die Sitzung und verabschiedet die Delegierten. Er dankt ihnen für ihr aktives und konstruktives Mitwirken. Sein Dank gilt ebenfalls dem Team des Generalsekretariats, welches die ÄK wiederum ausgezeichnet organisiert hat. Er wünscht allen eine gute Heimkehr. 


\section{Glossar}

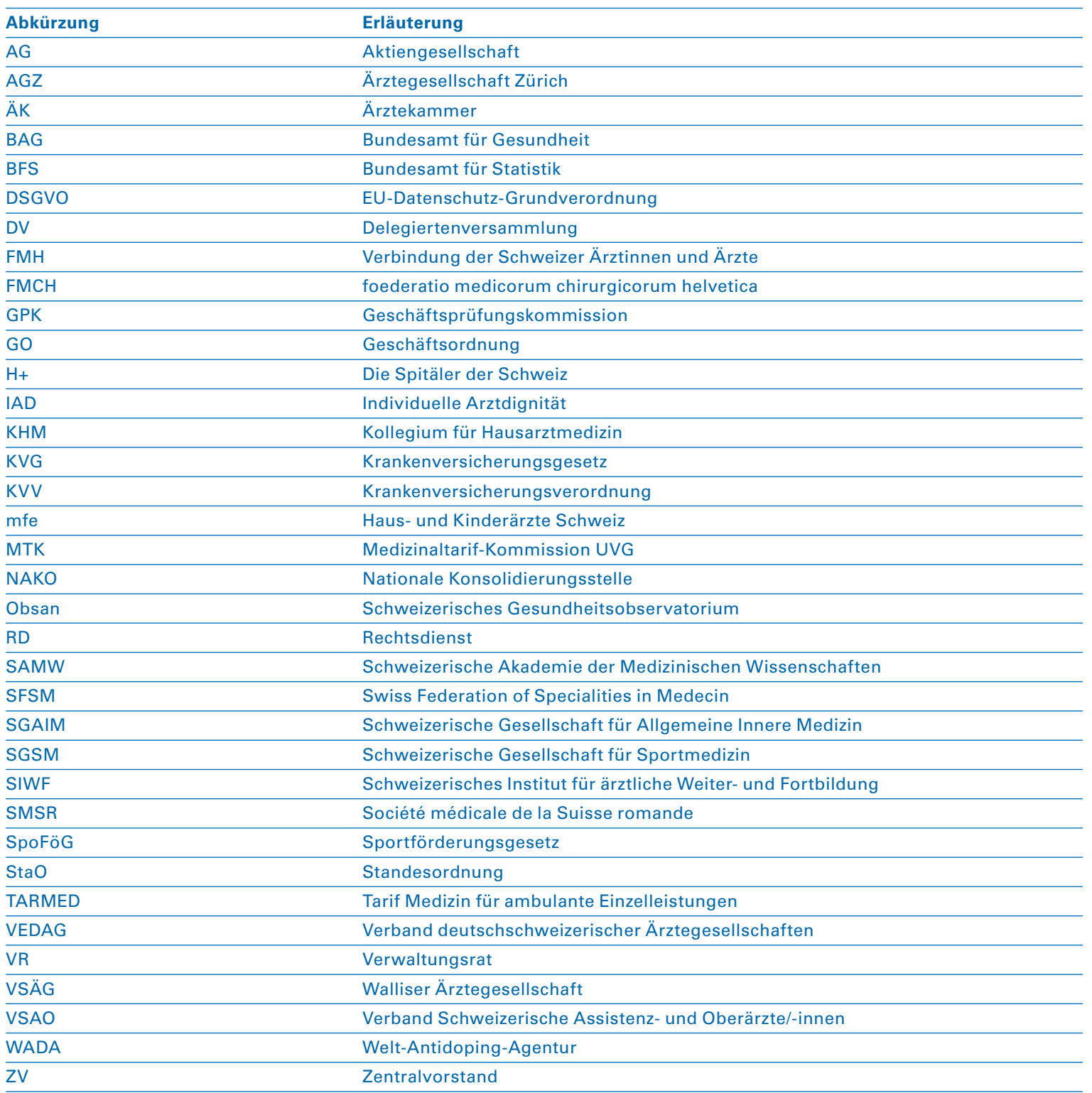

